\title{
Comparison of Resting-State Spontaneous Brain Activity between Treatment-Naive Patients with Schizophrenia and Obsessive-Compulsive Disorder
}

\section{Xiao-Man Yu}

Department of Psychiatry, the Affiliated Wuxi Mental Health Center of Nanjing Medical University, Wuxi, Jiangsu 214151, People's Republic of China

\section{Lin-Lin Qiu}

Anhui Medical University

\section{Hai-Xia Huang}

East China Sanatorium

\section{Xiang Zuo}

\section{East China Sanatorium}

\section{Zhen-He Zhou}

Department of Psychiatry, the Affiliated Wuxi Mental Health Center of Nanjing Medical University, Wuxi, Jiangsu 214151, People's Republic of China

\section{Shuai Wang}

Department of Psychiatry, the Affiliated Wuxi Mental Health Center of Nanjing Medical University, Wuxi, Jiangsu 214151, People's Republic of China

Hai-Sheng Liu

Department of Psychiatry, the Affiliated Wuxi Mental Health Center of Nanjing Medical University, Wuxi, Jiangsu 214151, People's Republic of China

\section{Lin Tian ( $\nabla$ tianz@njmu.edu.cn )}

Department of Psychiatry, the Affiliated Wuxi Mental Health Center of Nanjing Medical University, Wuxi, Jiangsu 214151, People's Republic of China

\section{Research Article}

Keywords: schizophrenia, obsessive-compulsive disorder, resting-state fMRI, amplitude of low-frequency fluctuation, degree centrality, regional homogeneity

Posted Date: April 21st, 2021

DOI: https://doi.org/10.21203/rs.3.rs-410695/v1 
License: (c) (i) This work is licensed under a Creative Commons Attribution 4.0 International License. Read Full License 


\section{Comparison of Resting-State Spontaneous Brain Activity between Treatment-Naive Patients with Schizophrenia and Obsessive-Compulsive Disorder}

4 Xiao-Man Yu ${ }^{1 \dagger}$, Lin-Lin Qiu ${ }^{2,3 \dagger}$, Hai-Xia Huang ${ }^{4}$, Xiang Zuo ${ }^{4}$, Zhen-He Zhou ${ }^{{ }^{*}}$,

5 Shuai Wang', Hai-Sheng Liu' ${ }^{1}$, and Lin Tian ${ }^{*}$ *

$6{ }^{1}$ Department of Psychiatry, the Affiliated Wuxi Mental Health Center of Nanjing

7 Medical University, Wuxi, Jiangsu 214151, People's Republic of China;

$8{ }^{2}$ School of Mental Health and Psychological Sciences, Anhui Medical University, Hefei,

9 Anhui 230032, People's Republic of China;

$10{ }^{3}$ Anhui Province Key Laboratory of Cognition and Neuropsychiatric Disorders \&

11 Collaborative Innovation Center of Neuropsychiatric Disorders and Mental Health,

12 Hefei, Anhui 230032, People's Republic of China;

$13{ }^{4}$ Department of Medical imaging, East China Sanatorium, Wuxi, Jiangsu 214100,

14 People's Republic of China;

15 These authors contributed equally to this work.

16 *Correspondence:

17 Lin Tian; Zhen-He Zhou

18 tianz@njmu.edu.cn; zhouzh@njmu.edu.cn 
19 Background: Schizophrenia (SZ) and Obsessive-compulsive disorder (OCD) share

20 many demographic and clinical symptoms, genetic risk factors, pathophysiological

21 underpinnings, and brain structure and function. However, the differences in the

22 spontaneous brain activity patterns between the two diseases remain unclear. Here this

23 study aimed to compare the features of intrinsic brain activity in treatment-naive

24 patients with schizophrenia (SZ) and obsessive-compulsive disorder (OCD) and to

25 explore the relationship between spontaneous brain activity and the severity of

26 symptoms.

27 Methods: Twenty-two treatment-naive patients with SZ, twenty-seven treatment-naive

28 patients with $\mathrm{OCD}$, and sixty healthy controls underwent resting-state functional

29 magnetic resonance imaging (fMRI). The amplitude of low-frequency fluctuation

30 (ALFF), regional homogeneity (ReHo) and degree of centrality (DC) of SZ group, OCD

31 group and healthy control (HC) group were compared.

32 Results: Compared with SZ group and HC group, patients with OCD had significantly

33 higher ALFF in the right angular gyrus and the left middle frontal gyrus/precentral

34 gyrus, and significantly lower ALFF in the left superior temporal gyrus/insula/rolandic

35 operculum and the left postcentral gyrus. Compared with HC group, lower ALFF values

36 in the right supramarginal gyrus/inferior parietal lobule and DC values of the right 2 
37 lingual gyrus/calcarine fissure and surrounding cortex of the two patient groups, higher

38 ReHo values in OCD group and lower ReHo values in SZ group in the right angular

39 gyrus/middle occipital gyrus brain region, and higher DC values in the right inferior

40 parietal lobule/angular gyrus in SZ group were documented in the present study. In

41 addition, the ALFF values of the left postcentral gyrus were positively correlated with

42 positive subscale score and general psychopathology subscale score respectively on the

43 Positive and Negative Syndrome Scale (PANSS) in SZ group. The ALFF values in the

44 left superior temporal gyrus/insula/rolandic operculum of patients with OCD were

45 positively correlated with compulsion subscale score and total score respectively on the

46 Yale-Brown Obsessive-Compulsive Scale (Y-BOCS).

47 Conclusion: Our data showed various patterns of spontaneous brain activity damage in

48 resting-state between treatment-naive patients with SZ and OCD, which might imply

49 different underlying neurobiological mechanisms in SZ and OCD.

50 Keywords: schizophrenia, obsessive-compulsive disorder, resting-state fMRI,

51 amplitude of low-frequency fluctuation, degree centrality, regional homogeneity 


\section{Background}

53 Although Schizophrenia (SZ) and obsessive-compulsive disorder (OCD) are separate

54 diagnostic entities, they both share high comorbidity, and the family history of OCD is

55 a risk factor for SZ, suggesting that they may have some common neurobiological bases

56 [1]. SZ and OCD equally belong to neurodevelopmental disorders and are characterized

57 by similar traits, e.g., reportedly numerous overlaps between the two disorders in some

58 domains, like demographic and clinical characteristics, genetic risk factors,

59 pathophysiological underpinnings, and brain structure and function [2, 3].

60 There is increasing evidence that SZ and OCD share neurobiological abnormalities [4,

$615]$, but some studies have failed to find overlap between them. For example, some

62 studies have found that compared with OCD, SZ represents more serious biological

63 disorders and greater neurological abnormalities [6-8]. It was found that there were

64 abnormalities in brain structure and brain function activity in patients with SZ and OCD

65 compared to those in healthy controls [9-12]. However, the unique and shared

66 neuroanatomical characteristics of the two diseases have not been fully identified [2, 5,

67 8]. To address the issue, there have been increasing research proposals to directly

68 compare the brain imaging characteristics between SZ and OCD under the same 
69 research methodology and framework, which is conducive to a better understanding of

70 the relationship between the two disorders $[5,8,13,14]$.

71 Resting-state functional magnetic resonance imaging (rs-fMRI) is a promising tool

72 for examining the blood oxygen level-dependent (BOLD) signal of the spontaneous

73 fluctuation of the whole brain, which does not require subjects to participate in

74 cognitive activities and is more convenient in clinical practice $[15,16]$. Several methods

75 such as the amplitude of low-frequency fluctuation (ALFF), regional homogeneity

76 (ReHo), and degree centrality (DC) have been proposed to explore spontaneous brain

77 activity in local and distant brain regions. These three values complement each other

78 and define brain functional characteristics from different perspectives [17-23]. ALFF is

79 an indicator that is used to detect the regional intensity of spontaneous fluctuation in

80 the BOLD signal, which pinpoints the spontaneous neural activity of a specific region

81 and physiological state of the brain in a resting state $[17,19]$. The ReHo method, testing

82 the local correlations in BOLD time series by using Kendall's coefficient of

83 concordance $(\mathrm{KCC})$, is often used to investigate regional synchronizations of temporal

84 changes in the brain. A higher ReHo value for a given brain region indicates greater

85 regional coherence $[20,21]$. Based on the voxel level, DC is a measure of the

86 connectome graph indexing the number of direct connections for a given node and 
87 reflects its functional connectivity (FC) within the whole brain network without

88 requiring a priori selection $[22,23]$. A node with high DC indicates that it has many

89 direct connections to other nodes in the brain networks. In recent years, these three

90 values have been widely used to investigate functional modulations in numerous

91 neuropsychiatric disorders [23-28].

92 Previous studies have found that patients with OCD showed a variety of

93 impairments, such as executive function, visuospatial and verbal memory, and

94 emotional processing [9, 10]. Neurobiologically, these functional defects are

95 considered to be related to functional abnormalities within not only the cortico-striato-

96 thalamo-cortical (CSTC) circuits, but also more extensive local brain outside the CSTC

97 circuits, e.g., the prefrontal cortex, parietal cortex, occipital cortex, and other brain

98 regions $[9,10,23,29,30]$. Patients with $\mathrm{SZ}$ can be involved in visual, auditory, and

99 other sensory impairments and cognitive impairments [31, 32]. It is believed that

100 schizophrenia has a complex etiopathogenesis and is related to a wide range of neural

101 circuits [33]. A meta-analysis reported that patients with SZ had abnormal brain

102 function in a wide range of brain regions such as frontal, temporal, parietal, occipital,

103 and orbitofrontal cortices [34]. As for fMRI studies, decreased FC in distinct occipital

104 subdivisions were reported by a recent resting-state study in patients with OCD [10] 
105 and increased activation in the bilateral dorsolateral prefrontal cortex (dlPFC), as well

106 as a decreased activation in the left inferior occipital gyrus (IOG), were demonstrated

107 in executive function and emotional processing fMRI studies by a recent meta-analysis

108 [9]. In addition, previous fMRI studies have shown that the ALFF is lower in people

109 with either SZ [27, 35, 36] or OCD [37-39], and the ReHo is impaired in people with

110 either SZ [40] or OCD [41]. Also, the DC declines in people with SZ [42, 43] or

$111 \mathrm{OCD}[44,45]$. Moreover, SZ and OCD may putatively share common neural networks

112 in several crucial regions, including the anterior cingulate gyrus, orbitofrontal cortex

113 (OFC) and thalamus, based on the review of several previous researches [46].

114 Particularly, under the same research conditions, our previous diffusion MRI study

115 showed that SZ and OCD had different patterns of anatomical and topological

116 organizations, which both present more severe and extensive disruptions in SZ [8].

117 Taken together, associations of brain networks between patients with SZ and OCD have

118 been proposed and furthermore many previous fMRI studies have demonstrated

119 abnormalities of ALFF, ReHo, and DC in multiple brain regions of patients with either

$120 \mathrm{SZ}$ or OCD, whereas no consistent conclusion has been reached.

121 However, as far as we know, there have been very few studies combining multiple

122 values to directly compare intrinsic brain abnormalities between SZ and OCD. 
123 Therefore, in the present study, we aimed to compare the characteristics of resting-state

124 spontaneous brain activity between treatment-naive patients with SZ and OCD by

125 adopting ALFF, ReHo and DC, and further to explore the relationships between brain

126 spontaneous activities and clinical symptoms. We hypothesized that both SZ and OCD

127 have abnormal spontaneous neural activity, whereas they share distinct neural activity.

128 This study was to compare the SZ mechanism with the OCD mechanism in ALFF,

129 ReHo and DC.

130 Methods

\section{Participants}

132 This study was approved by the Research Ethics Review Board of Wuxi Mental Health

133 Center. All methods were performed in accordance with the relevant guidelines and

134 regulations, and all participants provided written informed consent. We collected

135 imaging data of 29 patients with SZ, 29 patients with OCD and 65 healthy controls

136 (HC). All patients met the DSM-IV-TR criteria and none of them had received any

137 pharmacologic treatment or psychotherapies before the MRI scanning of this study.

138 MRI scans and evaluations of clinical symptoms of the patients were completed on the

139 same day. Positive and Negative Syndrome Scale (PANSS) was conducted in patients 
140 with SZ by experienced psychiatrists. As for patients with OCD, the severity of

141 obsessive-compulsive symptoms, anxious and depressive symptoms were respectively

142 assessed by Yale-Brown Obsessive Compulsive Scale (Y-BOCS) [47], Hamilton

143 Anxiety Rating Scale (HARS) [48] and 24-item Hamilton Rating Scale for Depression

144 (24-HDRS) [49], respectively. Individuals having a lack of current or historic diagnoses

145 of any psychiatric disorder were chosen as healthy controls. Besides, individuals with

146 family histories of any psychiatric disorders or neurological illnesses were excluded

147 from healthy controls. All recruited participants were right-handed when assessed with

148 Edinburgh Handedness Inventory [50]. Besides, exclusion criteria for all participants

149 include brain injury, intracranial pathology, substance abuse, neurological illness,

150 pregnancy, contraindications of MRI, and head movements during scanning more than

$1513 \mathrm{~mm}$ or $3.0^{\circ}$ in any direction. Four patients with SZ and 1 healthy control were

152 excluded because of incomplete collection function. Three patients with SZ, 2 patients

153 with OCD, and 4 healthy controls were excluded due to excessive head motion

154 cumulatively more than $3 \mathrm{~mm}$ or $3^{\circ}$ in any direction. Finally, 22 patients with SZ, 27

155 patients with OCD, and 60 healthy controls were included in the statistical analysis.

156 Table 1 provides detailed demographic and clinical characteristics.

\section{Data acquisition}


158 MRI was performed at the Department of Medical Imaging, Wuxi People's Hospital,

159 Nanjing Medical University by using a 3.0-Tesla Magnetom Trio Tim (Siemens

160 Medical System, Erlangen, Germany) and a 12-channel phased-array head coil. All

161 participants, whose heads were fixed with foam pads to reduce scanner noise and head

162 motion, were required to close their eyes, to relax their minds but not to fall asleep, and

163 to move as little as possible during imaging acquisition. Three-dimensional $\mathrm{T}_{1}$ -

164 weighted images were acquired using the 3D magnetization-prepared rapid acquisition

165 gradient-echo sequence with the following parameters: time repetition $(\mathrm{TR})=2530 \mathrm{~m}$

$166 \mathrm{~s}$, time echo $(\mathrm{TE})=3.44 \mathrm{~ms}$, flip angle $=7^{\circ}$, field of view $(\mathrm{FOV})=256 \times 256 \mathrm{~mm} 2$,

167 matrix size $=256 \times 256$, slice thickness $=1 \mathrm{~mm}, 192$ sagittal slices, acquisition voxel

168 size $=1 \times 1 \times 1 \mathrm{~mm} 3$, total acquisition time $=649 \mathrm{~s}$. Resting-state fMRI was conducted

169 by using a gradient-echo planar imaging sequence. $\mathrm{T}_{2}$-weighted images were taken

170 (single shot, $\mathrm{TR}=2000 \mathrm{~ms}, \mathrm{TE}=30 \mathrm{~ms}$, flip angle $=90, \mathrm{FOV}=220 \times 220 \mathrm{~mm}^{2}$, matrix

171 size $=64 \times 64$, slice thickness $=4 \mathrm{~mm}, 33$ axial slices, acquisition voxel size $=3.4 \times$

$1723.4 \times 4 \mathrm{~mm}^{3}$, resulting in 240 volumes).

\section{Data preprocessing}

174 Analysis of the RS-fMRI data was performed by using Data Processing and Analysis

175 of Brain Imaging [51] (DPABI; http://rfmri.org/DPABI_V4.3) in MATLAB 2013b (The 
176 Math Works, Natick, MA, USA) based on Statistical Parametric Mapping (SPM12;

177 http://www.fil.ion.ucl.ac.uk/spm/software/spm12). The first 10 time points were

178 discarded for initial signal stabilization. The remaining 230 volumes were corrected for

179 the intra-volume acquisition time delay using slice timing correction and were realigned

180 for head movement correction. If the head movement was more than $3 \mathrm{~mm}$ or $3^{\circ}$, the

181 data were excluded from the analysis. To further eliminate the residual effect of motion

182 on resting-state fMRI measurement, Jenkinson's mean framewise displacement (mean

183 FD) was calculated based on their realignment parameters to quantify head motion,

184 which was used as a covariable of all voxel-wise group functional analyses [17, 52].

185 Each $\mathrm{T}_{1}$-weighted image was registered with the average functional image, and the

186 image was divided into white matter, gray matter, and cerebrospinal fluid tissue maps.

187 Then the image space was normalized to the standard Montreal Neurological Institute

188 (MNI) space, and the resampling was $3 \times 3 \times 3 \mathrm{~mm}^{3}$. Subsequently, the generalized

189 linear model was used to regress the signals from white matter and cerebrospinal fluid

190 and the covariates of Friston-24 parameters, and linear trends of the time courses were

191 removed from the fMRI data [53, 54]. Before ALFF analysis, a Gaussian filter (6-mm

192 full-width half-maximum, FWHM) was used for spatial smoothing, but smoothing was

193 performed after ReHo and DC calculations. Smoothing before the calculation of ReHo 
194 and DC will cause the regional correlation of adjacent voxels and affect the calculation

195 of the above two values, so smoothing was usually carried out after calculation to

196 reduce spatial noise and reduce the incompleteness of the registration effect of the

197 subjects [22, 55]. Finally, DPABI_V4.3 was used to calculate the values of the ALFF,

198 ReHo and DC.

199 ALFF analysis

200 After data preprocessing, the time series for each voxel was transformed to the

201 frequency domain using fast Fourier transforms, and the square root of this spectrum

202 was calculated for each frequency and then averaged across $0.01-0.08 \mathrm{~Hz}$ [19]. This

203 averaged square root was used as an ALFF index. For standardization, the ALFF of

204 each voxel was divided by the global mean ALFF, to get the mALFF map [56, 57].

\section{ReHo and DC analyses}

206 ReHo and DC values were measured based_on unsmoothed data. After preprocessing,

207 a temporal filter $(0.01-0.08 \mathrm{~Hz})$ was applied to reduce the influences of high-frequency

208 physiological noises and low-frequency drifts.

209 The ReHo value was obtained on a voxel-by-voxel basis by calculating Kendall's

210 coefficient of concordance of a given voxel with those of its 26 nearest neighbors [20]. 
211 Then the ReHo value of each voxel was divided by the global mean ReHo of each

212 individual, to get the mReHo map [56, 57]. Next, mReHo maps were smoothed with a

213 6-mm FWHM Gaussian kernel.

214 After data preprocessing, fMRI data were used to calculate the voxel-wise DC,

215 and then Pearson's correlation method was utilized to correlate the time series of each

216 voxel with the time series of every other voxel, after which a matrix of Pearson's

217 correlation coefficients matrix was obtained. Next, the correlation coefficient of $r=0.25$

218 was used as the lowest threshold to eliminate the low time correlation caused by signal

219 noise [22, 58]. Subsequently, the binary degree centrality values of the whole-brain

220 network were calculated [57, 59]. As a result, each participant obtained a map of the

221 DC value of each gray matter voxel. Before group-level statistical analysis, we divided

222 DC of each voxel by the global mean DC, to get the mDC map, and then used Gaussian

223 smoothing kernels (full width half maximum, half-width $=6 \mathrm{~mm}$ ) to spatially smooth

224 all individual mDC maps [56, 57].

\section{Statistical analyses}

226 The demographic and clinical data of the subjects were analyzed by SPSS 25.0 software

227 (SPSS, Chicago, IL, United States). The normal distribution data were described as the 
228 average \pm standard deviation, while the non-normal distribution data were presented by

229 the median (the first quartile-the third quartile). The age and education level of the three

230 groups showed normal distribution, and then a one-way analysis of variance (ANOVA)

231 was used to test differences among the three groups. The course of disease of the two

232 patient groups was not subject to the normal distribution, and a Mann-Whitney U test

233 was used to assess between-group differences. The mean framewise displacement (FD)

234 was also not subject to the normal distribution, and a Kruskal-Wallis test was used to

235 detect whether there were significant differences among the three groups. A $P$ value of

$236<0.05$ was considered to be statistically significant.

237 The statistical analysis of fMRI data was conducted using SPM12 software. The

238 voxel-wise ANCOVA was used to test the differences in ALFF, ReHo and DC among

239 the three groups. The confounding factors of age, sex, the level of education, and

240 Jenkinson's mean FD were controlled as covariates. The multiple comparisons

241 correction of statistical F-maps was performed with family-wise error (FWE) cluster-

242 corrected $(P<0.05)$ when using a primary voxel determining the threshold of $P<0.001$

243 to protect against false-positive findings. For the clusters showing significant

244 differences among the three groups, the mean ALFF, ReHo and DC values were

245 extracted from the cluster for each participant. Then the post-hoc analyses were 
246 conducted using SPSS25.0, and the analyses were corrected for multiple comparisons

247 using Bonferroni correction at a statistical significance level of $P<0.05$. Moreover,

248 partial correlation analysis was performed to evaluate the relationship between the

249 ALFF, ReHo and DC values extracted from the above-mentioned significant difference

250 clusters respectively and the severity of symptoms (Y-BOCS and PANSS scores). Age,

251 sex, the level of education, Jenkinson's mean FD, and course of disease were taken as

252 covariates. Results with $P<0.05$ (uncorrected) were considered statistically significant.

\section{Results}

\section{Demographics and clinical characteristics}

255 Demographic, clinical variables and the mean FD of the participants are presented in

256 Table 1. There was no significant difference in the mean FD among SZ group, OCD

257 group and HC group $(P>0.05)$. There were significant differences in age and education

258 level among the three groups $(P<0.05)$, but not in $\operatorname{sex}(P>0.05)$. The results of post-

259 hoc analysis showed that the age of OCD group was lower than that of HC group, and

260 the education level of SZ group was lower than that of HC group (Bonferroni, $P<0.05$ ).

261 The duration of disease in OCD group was significantly longer than that in SZ group

$262(P<0.05)$ 
264 The results of ANCOVA analysis showed that there were significant differences among

265 the three groups (voxel significance, $P<0.001$; cluster significance, $P<0.05$, FWE

266 correction) in the right angular gyrus, the left superior temporal gyrus/insula/rolandic

267 operculum, the left middle frontal gyrus/ precentral gyrus, the left postcentral gyrus,

268 and the right supramarginal gyrus/inferior parietal lobule (Figure 1A; Table 2).

269 Post hoc t-tests $(P<0.05$, Bonferroni correction) showed that compared to HC

270 group, the ALFF values in the right supramarginal gyrus/inferior parietal lobule of the

271 two patient groups were lower (SZ group: 1.07 \pm 0.18 ; OCD group: $1.12 \pm 0.15$; Healthy

272 group: $1.37 \pm 0.29$, Figure 2A). Compared to HC group, patients with OCD had higher

273 ALFF in the right angular gyrus (SZ group: $1.08 \pm 0.26$; OCD group: $1.60 \pm 0.48$; Healthy

274 group: $1.17 \pm 0.21$, Figure 2A) and the left middle frontal gyrus/precentral gyrus (SZ

275 group: $0.75 \pm 0.10$; OCD group: $0.92 \pm 0.20$; Healthy group: $0.68 \pm 0.12$, Figure $2 \mathrm{~A}$ ), and

276 lower ALFF in the left superior temporal gyrus/insula/rolandic operculum (SZ group:

277 0.76 \pm 0.16 ; OCD group: $0.60 \pm 0.11$; Healthy group: $0.75 \pm 0.14$, Figure $2 \mathrm{~A}$ ) and the left

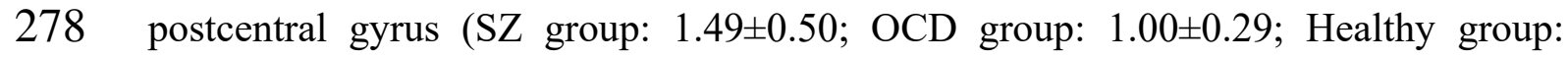

$2791.28 \pm 0.40$, Figure 2A). Compared to OCD group, the ALFF values of SZ group in the

280 left superior temporal gyrus/insula/rolandic operculum and the left postcentral gyrus 
281 were significantly higher, while those in the right angular gyrus and the left middle

282 frontal gyrus/precentral gyrus were significantly lower (Figure 2A).

\section{ReHo differences among the three groups}

284 As for the ReHo, ANCOVA showed significant differences in the right angular

285 gyrus/middle occipital gyrus (voxel significance, $P<0.001$; cluster significance, $P<$

286 0.05, FWE correction) (Figure 1B; Table 2) among the three groups. Compared with

287 HC group, the ReHo values of the right angular gyrus/middle occipital gyrus (SZ

288 group:1.23 \pm 0.20 ; OCD group:1.47 \pm 0.17 ; Healthy group: $1.32 \pm 0.11$, Figure 2B) were

289 significantly higher in OCD group, whereas those were significantly lower in SZ group

$290(P<0.05$, Bonferroni correction $)$.

291 DC differences among the three groups

292 Analysis of ANCOVA showed that there were significant differences in DC of the right

293 lingual gyrus/calcarine fissure and surrounding cortex and the right inferior parietal

294 lobule/angular gyrus among the three groups (Figure 1C; Table 2). Compared to HC

295 group, the DC values in the right inferior parietal lobule/angular gyrus (SZ group:

$2961.03 \pm 0.21$; OCD group: $0.83 \pm 0.21$; Healthy group: $0.76 \pm 0.18$, Figure 2C) were

297 significantly higher in SZ group and significantly lower in the right lingual 
298 gyrus/calcarine fissure and surrounding cortex (SZ group: 1.21 \pm 0.28 ; OCD group:

299 1.28 \pm 0.28 ; Healthy group: $1.51 \pm 0.22$, Figure 2C) in both SZ and OCD group.

300 Compared to OCD group, the DC values of SZ group were significantly higher in the

301 right inferior parietal lobule/angular gyrus $(P<0.05$, Bonferroni correction).

\section{Correlation with clinical scores}

303 In SZ group, the ALFF values in the left postcentral gyrus were positively correlated

304 with PANSS positive subscale score $(\mathrm{r}=0.588, P=0.013$, uncorrected, Figure 3A $)$ and

305 PANSS general psychopathological subscale score $(\mathrm{r}=0.488, P=0.047$, uncorrected,

306 Figure 3A), respectively. The ALFF values in the left superior temporal

307 gyrus/insula/rolandic operculum of patients with OCD were positively correlated with

308 compulsion subscale score $(\mathrm{r}=0.463, P=0.030$, uncorrected, Figure 3B) and total

309 score of Y-BOCS $(r=0.5713, P=0.005$, uncorrected, Figure 3B).

\section{Discussion}

311 To the best of our knowledge, this work is the first attempt to directly compare the

312 intrinsic brain functional alterations in treatment-naive patients with SZ and OCD by

313 adopting multiple imaging parameters. Compared to using any one of the parameters

314 alone, the combination of the three methods may yield a valid and comprehensive 
315 pathophysiological framework in brain studies [60, 61]. In the present study, there were

316 significant differences among healthy controls, patients with SZ and OCD respectively

317 in the values of ALFF, ReHo and DC in a series of brain regions. Our results showed

318 that compared with patients with SZ and HC, patients with OCD had significantly

319 higher ALFF in the right angular gyrus and the left middle frontal gyrus/precentral

320 gyrus, and significantly lower ALFF in the left superior temporal gyrus/insula/rolandic

321 operculum and the left postcentral gyrus, but there was no significant difference in

322 ALFF of the above-mentioned brain regions between SZ group and HC group.

323 Compared to HC group, the ALFF values in the right supramarginal gyrus/inferior

324 parietal lobule of the two patient groups were lower. The ReHo values of the right

325 angular gyrus/ middle occipital gyrus in OCD group were significantly higher than

326 those in HC group, while those in SZ group were significantly lower than those in HC

327 group. Compared with HC group, the DC values in the right inferior parietal

328 lobule/angular gyrus were significantly higher in SZ group, and significantly lower in

329 the right lingual gyrus/calcarine fissure and surrounding cortex in both SZ and OCD

330 group. The exploratory correlation analyses showed that the ALFF values of specific

331 brain regions were related to the clinical symptoms of the patients with SZ and OCD

332 respectively. 
333 ALFF alterations in patients with SZ and OCD

334 ALFF was supposed to reflect the intensity of regional spontaneous neuronal activity

335 [62]. Previous studies have found that patients with OCD generally have abnormalities

336 in the brain areas within the cortico-striato-thalamo-cortical (CSTC) circuits, whereas

337 increasing abnormalities have also been found in the brain regions outside the CSTC

338 circuits. This study found that patients with OCD displayed abnormal spontaneous

339 neural activities in the brain regions outside the CSTC circuits, such as the parietal lobe,

340 temporal lobe, occipital lobe, and prefrontal lobe, which was consistent with the results

341 of previous studies $[23,28,30]$. The results of this study did not demonstrate the

342 damage of spontaneous brain activities in the common CSTC circuits such as the

343 orbitofrontal lobe, thalamus, and anterior cingulate gyrus in patients with OCD, which

344 may be related to the difference of sample size and research methods. Our findings

345 provide broader evidence that brain regions outside the CSTC circuits are involved in

346 the pathophysiology of OCD.

347 The inferior parietal lobule, including the supramarginal gyrus and angular gyrus,

348 is a major network hub of the human brain and plays an important role in a wide range

349 of behaviors and functions from bottom-up perception to social cognition. It plays an

350 important role in many networks, including frontoparietal control network, default 20 
351 mode network (DMN), ventral attention network, and cingulo-opercular network.

352 Several studies have reported changes in connectivity in these networks in patients with

353 OCD $[63,64]$. It was reported that patients with OCD have abnormalities in the inferior

354 parietal lobule, reflecting a lack of cognitive flexibility, which may be associated with

355 the repetition of obsessive-compulsive symptoms and behaviors [65]. Previous reviews

356 suggested that the inferior parietal lobule may play an important role in SZ [66]. The

357 impairment of the inferior parietal lobule in patients with SZ mainly affects their body

358 image, sensory integration, self-concept, and executive function [66]. We observed that

359 the ALFF values in the right supramarginal gyrus/inferior parietal lobule of the two

360 patient groups were lower than those in HC group, and the ALFF values in the right

361 angular gyrus in patients with OCD were significantly higher than those in HC group,

362 suggesting that the local spontaneous brain activities in the inferior parietal lobule were

363 impaired in both patient groups.

364 ReHo alterations in patients with SZ and OCD

365 ReHo reflects neural functional synchronization in local brain regions [20, 67].

366 Compared with HC group, the ReHo values of the right angular gyrus/middle occipital

367 gyrus were significantly higher in OCD group and lower in SZ group. The angular gyrus

368 is located in the parietal lobe and is mainly related to skill learning, attention, and 21 
369 working memory [68]. These present findings are compatible with the previous

370 functional alterations results. Niu et al [69]. demonstrated that higher ReHo values were

371 found in the left angular gyrus in patients with OCD. Nierenberg et al [70]. found that

372 the volume of the left angular gyrus in patients with new-onset schizophrenia was

373 smaller than that in healthy subjects and proposed that the angular gyrus may be the

374 neuroanatomical substrate of the expression of SZ. The occipital cortex is considered

375 to play an important role in early visual processing, such as visual hallucinations and

376 object-recognition defects [71]. Fan et al. found that patients with OCD had higher

377 ALFF in the right middle occipital gyrus [72]. Moreover, the occipital cortex was also

378 demonstrated to play an important role in OCD by several previous studies [72, 73]. Yu

379 et al [74]. reported lower ReHo in the occipital lobe in patients with SZ. Therefore, the

380 distinct patterns of ReHo values in the right middle occipital gyrus, which were lower

381 in patients with SZ and higher in patients with OCD, respectively, may indicate

382 differently impaired visual processing in the two different diseases, which is compatible

383 with the previous findings on the eye movements characteristics of patients with SZ

384 and OCD [75].

385 DC alterations in patients with SZ and OCD 
386 DC reflects the role and importance of the nodes or brain regions in the brain networks

$387[22,23]$. Both patients' groups showed lower DC in the right lingual gyrus/calcarine

388 fissure and surrounding cortex in the current study. The lingual gyrus and calcarine

389 fissure and surrounding cortex are located in the occipital lobe and are closely related

390 to visual information processing. The lingual gyrus, an important part of the visual

391 recognition network, plays a role in mediating visual word processing and analyzing

392 the complex features of visual forms, and participates in emotion perception during

393 facial stimulation, especially facial recognition [76]. Several studies have observed

394 structural or functional abnormalities of the lingual gyrus or occipital lobe in patients

395 with SZ and OCD [9, 77-80]. The meta-analysis of Gao et al. [77] showed structural

396 abnormalities in the lingual gyrus in drug-free patients with SZ. Moreira et al. [9]

397 observed that patients with OCD displayed reduced functional connectivity within and

398 between visual and sensorimotor networks. The authors of the present study believe

399 that these results are consistent with the hypothesis that occipital/parietal deactivation

400 is one of the mechanisms of OCD phenotype. Based on this current study, we speculate

401 that the lower DC in the right lingual gyrus/calcarine fissure and surrounding cortex

402 may contribute to the visual processing deficits in SZ and OCD. This study also found

403 that the DC values of the right inferior parietal lobule/angular gyrus in SZ group were 
404 significantly higher than those in HC group, whereas there was no significant difference

405 in DC value of these brain areas between OCD group and HC group. We speculate that

406 the resting state of the inferior parietal lobule in Patients with SZ is highly vigilant,

407 which may be attributed to the impairment of executive function and cognitive function.

408 Among these present findings, the abnormalities of ALFF, ReHo and DC in the

409 angular gyrus were especially noticeable. Recent researches have suggested that the

410 angular gyrus is responsible for complex mental phenomena and processes, such as

411 understanding visual and audio inputs [81], interpreting languages [82], retrieving

412 memories [83], and maintaining consciousness [84]. Moreover, the angular gyrus has

413 been demonstrated to be one of the overlapping regions between the default mode

414 network and social brain networks [85]. Schilbach et al. [85] proposed that the

415 physiological "baseline" of the human brain is linked to the psychological "baseline",

416 the predisposition human beings have for considering social cognition as the default

417 mode of thought. Concerning SZ and OCD, previous studies have revealed that the

418 functional integration with the right angular gyrus was significantly correlated with

419 sustained attention in both SZ and OCD patient samples [86]. Our data provide

420 empirical evidence about the angular gyrus, whose abnormal spontaneous activities

421 may play an important role in the pathophysiological mechanisms of SZ and OCD. 
422 The positive correlation between ALFF and clinical symptoms severity in patients with

$423 \quad$ SZ and OCD

424 Our findings showed that the ALFF values in the left postcentral gyrus were positively

425 associated with the severity of clinical symptoms expressed by positive subscale score

426 and general psychopathological subscale score on the PANSS in patients with SZ, and

427 the values of ALFF in the left superior temporal gyrus, insula, and rolandic operculum

428 were positively associated with the severity of clinical symptoms presented by

429 compulsion subscale score and total score on the Y-BOCS in patients with OCD. These

430 present findings are compatible with the previous studies. Qiu et al. [87] reported that

431 abnormal gray matter density in the left postcentral was found, and the abnormal gray

432 matter density in the left postcentral gyrus was associated with RSS, a specific eye

433 movement index of schizophrenia, which is related to the integration of several

434 perceptual/cognitive processes, including selective and sustained attention, and

435 working memory [88-90], reflecting the clinical hallucination severity of schizophrenia

436 [91]. As for OCD, the superior temporal gyrus was documented to be specifically

437 associated with social anhedonia in OCD [92]. Moreover, greater recruitment of the left

438 superior temporal gyrus was found in pediatric Patients with OCD than healthy controls 
439 during combined symptom provocation, which suggested the involvement of the

440 temporal poles in pediatric OCD during symptom provocation [93].

441 The limitations of our research

442 The present study has some potential limitations. First, the sample size used for imaging

443 analyses in this study is relatively small, which may limit the value of the research. For

444 example, due to the limitation of the sample size, difficulties in respectively subdividing

445 patients of SZ and OCD into different groups based on symptoms or subtypes increased,

446 resulting in a lack of full consideration of the heterogeneity of the sample. Second, we

447 carefully excluded the influence of confounding factors such as antipsychotic

448 medication in this study. However, the disadvantage is that the three groups of subjects

449 in our present study are unevenly matched in terms of the number of participants, age

450 and education level. In addition, it was reported that different standardized procedures

451 may affect the re-test reliability of ALFF, ReHo and DC [67]. Finally, the present

452 research is a cross-sectional study, and it's better to further adopt longitudinal studies

453 to explore the changes in patients' brain function before and after treatment based on

454 this study, which may be more helpful to the clinic.

455 Conclusions 
456 In summary, our data demonstrated various patterns of altered spontaneous brain

457 activities in the resting state between treatment-naive patients with SZ and OCD.

458 Moreover, the exploratory correlation analyses showed that the ALFF values of specific

459 brain regions were related to the clinical symptoms of patients with SZ and OCD

460 respectively. Convergent findings for abnormalities of the angular gyrus suggested this

461 brain region may play an important role in the pathophysiological mechanisms of SZ

462 and OCD. The distinct patterns of intrinsic brain activity were found in the two diseases

463 that are usually reported to share neurobiological abnormalities and genetic

464 characteristics, which may reveal that SZ and OCD have different underlying

465 neurobiological mechanisms.

466 Acknowledgments

467 The authors would like to thank all subjects for contributing their time and efforts to

468 this study.

\section{Abbreviations}

470 SZ: schizophrenia; OCD: obsessive-compulsive disorder; fMRI: resting-state

471 functional magnetic resonance imaging; ALFF: The amplitude of low-frequency

472 fluctuation; ReHo: regional homogeneity; DC: degree of centrality; HC: healthy control 
473 group; PANSS: Positive and Negative Syndrome Scale; Y-BOCS: Yale-Brown

474 Obsessive-Compulsive Scale; rs-fMRI: Resting-state functional magnetic resonance

475 imaging; BOLD: blood oxygen level-dependent; KCC: Kendall's coefficient of

476 concordance; FC: functional connectivity; CSTC: cortico-striato-thalamo-cortical;

477 dlPFC: dorsolateral prefrontal cortex; IOG: inferior occipital gyrus; OFC: orbitofrontal

478 cortex; HARS: Hamilton Anxiety Rating Scale; 24-HDRS: 24-item Hamilton Rating

479 Scale for Depression; TR: time repetition; TE: time echo; FOV: field of view; MNI:

480 Montreal Neurological Institute; FWHM: full-width half-maximum; mean FD:

481 Jenkinson's mean framewise displacement; FEW: family-wise error; ANOVA: one-way

482 analysis of variance; ANCOVA: Analysis of Covariance; SPSS: Statistical Package for

483 the Social Sciences; DMN: default mode network.

484 Authors' contributions

485 LT and ZZ designed the study. HH, XZ, SW, and HL contributed to the acquisition of

486 the data. XY, LQ, LT analyzed the data and drafted the manuscript. All authors

487 contributed to and have approved the final version for publication.

$488 \quad$ Funding 
489 This work was supported in part by China Natural Science Foundation (No. 81871081

490 and 81301148); the Special Foundation for Jiangsu Young Medical Talents (No.

491 QNRC2016175); Top Talent Support Program for young and middle-aged people of

492 Wuxi Health Committee and the Natural Science Foundation of the Colleges and

493 Universities in Anhui Province (No. KJ2019A0292). Sincere appreciation is extended

494 to the patients and control subjects for their valuable participation.

495 Availability of data and materials

496 The datasets used during the current study are available from the corresponding author

497 on reasonable request.

498 Ethics approval and consent to participate

499 This study was approved by the Medical Ethics Committee of Wuxi Mental Health

500 Center, Nanjing Medical University, China. All participants provided written informed

501 consent.

502 Consent for publication

503 Not applicable.

504 Competing interests 
505 The authors declare that they have no conflicts of interest in this work.

\section{Author details}

$507{ }^{1}$ Department of Psychiatry, the Affiliated Wuxi Mental Health Center of Nanjing

508 Medical University, Wuxi, Jiangsu 214151, People's Republic of China. ${ }^{2}$ School of

509 Mental Health and Psychological Sciences, Anhui Medical University, Hefei, Anhui

510 230032, People's Republic of China. ${ }^{3}$ Anhui Province Key Laboratory of Cognition

511 and Neuropsychiatric Disorders \& Collaborative Innovation Center of

512 Neuropsychiatric Disorders and Mental Health, Hefei, Anhui 230032, People's

513 Republic of China. ${ }^{4}$ Department of Medical imaging, East China Sanatorium, Wuxi,

514 Jiangsu 214100, People's Republic of China.

\section{References}

516 1. Meier SM, Petersen L, Pedersen MG, Arendt MC, Nielsen PR, Mattheisen M, Mors

517 O, Mortensen PB. Obsessive-compulsive disorder as a risk factor for schizophrenia: a

518 nationwide study. JAMA psychiatry. 2014; 71(11):1215-1221.

519 2. Poyurovsky M, Zohar J, Glick I, Koran LM, Weizman R, Tandon R, Weizman A.

520 Obsessive-compulsive symptoms in schizophrenia: implications for future psychiatric

521 classifications. Comprehensive psychiatry. 2012; 53(5):480-483. 
522 3. Narayanaswamy JC, Viswanath B, Veshnal Cherian A, Bada Math S, Kandavel T,

523 Janardhan Reddy YC. Impact of age of onset of illness on clinical phenotype in OCD.

524 Psychiatry research. 2012; 200(2-3):554-559.

525 4. Patel Y, Parker N, Shin J, Howard D, French L, Thomopoulos SI, Pozzi E, Abe Y,

526 Abé C, Anticevic A et al. Virtual Histology of Cortical Thickness and Shared

527 Neurobiology in 6 Psychiatric Disorders. JAMA psychiatry. 2021; 78(1):47-63.

528 5. Hawco C, Voineskos AN, Radhu N, Rotenberg D, Ameis S, Backhouse FA,

529 Semeralul M, Daskalakis ZJ. Age and gender interactions in white matter of

530 schizophrenia and obsessive compulsive disorder compared to non-psychiatric controls:

531 commonalities across disorders. Brain imaging and behavior. 2017; 11(6):1836-1848.

532 6. Ha TH, Yoon U, Lee KJ, Shin YW, Lee JM, Kim IY, Ha KS, Kim SI, Kwon JS.

533 Fractal dimension of cerebral cortical surface in schizophrenia and obsessive-

534 compulsive disorder. Neuroscience letters. 2005; 384(1-2):172-176.

535 7. Riffkin J, Yücel M, Maruff P, Wood SJ, Soulsby B, Olver J, Kyrios M, Velakoulis

536 D, Pantelis C. A manual and automated MRI study of anterior cingulate and orbito-

537 frontal cortices, and caudate nucleus in obsessive-compulsive disorder: comparison

538 with healthy controls and patients with schizophrenia. Psychiatry research. 2005;

539 138(2):99-113. 
540 8. Qin J, Sui J, Ni H, Wang S, Zhang F, Zhou Z, Tian L. The Shared and Distinct

541 White Matter Networks Between Drug-Naive Patients With Obsessive-Compulsive

542 Disorder and Schizophrenia. Frontiers in neuroscience. 2019; 13:96.

543 9. Moreira PS, Marques P, Magalhães R, Esteves M, Sousa N, Soares JM, Morgado

544 P. The resting-brain of obsessive-compulsive disorder. Psychiatry research

545 Neuroimaging. 2019; 290:38-41.

546 10. Picó-Pérez M, Moreira PS, de Melo Ferreira V, Radua J, Mataix-Cols D, Sousa N,

547 Soriano-Mas C, Morgado P. Modality-specific overlaps in brain structure and function

548 in obsessive-compulsive disorder: Multimodal meta-analysis of case-control MRI

549 studies. Neuroscience and biobehavioral reviews. 2020; 112:83-94.

550 11. van Erp TGM, Walton E, Hibar DP, Schmaal L, Jiang W, Glahn DC, Pearlson GD,

551 Yao N, Fukunaga M, Hashimoto R et al. Cortical Brain Abnormalities in 4474

552 Individuals With Schizophrenia and 5098 Control Subjects via the Enhancing Neuro

553 Imaging Genetics Through Meta Analysis (ENIGMA) Consortium. Biological

554 psychiatry. 2018; 84(9):644-654.

555 12. Arsalidou M, Yaple Z, Jurcik T, Ushakov V. Cognitive Brain Signatures of Youth

556 With Early Onset and Relatives With Schizophrenia: Evidence From fMRI Meta-

557 analyses. Schizophrenia bulletin. 2020; 46(4):857-868. 
558 13. White T, Langen C, Schmidt M, Hough M, James A. Comparative neuropsychiatry:

559 white matter abnormalities in children and adolescents with schizophrenia, bipolar

560 affective disorder, and obsessive-compulsive disorder. European psychiatry : the

561 journal of the Association of European Psychiatrists. 2015; 30(2):205-213.

562 14. Kwon JS, Shin YW, Kim CW, Kim YI, Youn T, Han MH, Chang KH, Kim JJ.

563 Similarity and disparity of obsessive-compulsive disorder and schizophrenia in MR

564 volumetric abnormalities of the hippocampus-amygdala complex. Journal of neurology,

565 neurosurgery, and psychiatry. 2003; 74(7):962-964.

566 15. Lee MH, Smyser CD, Shimony JS. Resting-state fMRI: a review of methods and

567 clinical applications. AJNR American journal of neuroradiology. 2013; 34(10):1866-

5681872.

569 16. Rosazza C, Minati L. Resting-state brain networks: literature review and clinical

570 applications. Neurological sciences : official journal of the Italian Neurological Society

571 and of the Italian Society of Clinical Neurophysiology. 2011; 32(5):773-785.

572 17. Zhang Z, Bo Q, Li F, Zhao L, Wang Y, Liu R, Chen X, Wang C, Zhou Y. Increased

573 ALFF and functional connectivity of the right striatum in bipolar disorder patients.

574 Progress in neuro-psychopharmacology \& biological psychiatry. 2020:110140. 
575 18. Gong J, Wang J, Luo X, Chen G, Huang H, Huang R, Huang L, Wang Y.

576 Abnormalities of intrinsic regional brain activity in first-episode and chronic

577 schizophrenia: a meta-analysis of resting-state functional MRI. Journal of psychiatry \&

578 neuroscience : JPN. 2020; 45(1):55-68.

579 19. Liu C, Xue Z, Palaniyappan L, Zhou L, Liu H, Qi C, Wu G, Mwansisya TE, Tao

$580 \mathrm{H}$, Chen $\mathrm{X}$ et al. Abnormally increased and incoherent resting-state activity is shared

581 between patients with schizophrenia and their unaffected siblings. Schizophrenia

582 research. 2016; 171(1-3):158-165.

583 20. Zang Y, Jiang T, Lu Y, He Y, Tian L. Regional homogeneity approach to fMRI data

584 analysis. NeuroImage. 2004; 22(1):394-400.

585 21. Ji L, Meda SA, Tamminga CA, Clementz BA, Keshavan MS, Sweeney JA,

586 Gershon ES, Pearlson GD. Characterizing functional regional homogeneity (ReHo) as

587 a B-SNIP psychosis biomarker using traditional and machine learning approaches.

588 Schizophrenia research. 2020; 215:430-438.

589 22. Zuo XN, Ehmke R, Mennes M, Imperati D, Castellanos FX, Sporns O, Milham MP.

590 Network centrality in the human functional connectome. Cerebral cortex (New York,

591 NY : 1991). 2012; 22(8):1862-1875. 
593 Abnormal functional connectivity of brain network hubs associated with symptom

594 severity in treatment-naive patients with obsessive-compulsive disorder: A resting-state

595 functional MRI study. Progress in neuro-psychopharmacology \& biological psychiatry.

596 2016; 66:104-111.

597 24. Chen J, Xu Y, Zhang K, Liu Z, Xu C, Shen Y, Xu Q. Comparative study of regional

598 homogeneity in schizophrenia and major depressive disorder. American journal of

599 medical genetics Part B, Neuropsychiatric genetics : the official publication of the

600 International Society of Psychiatric Genetics. 2013; 162b(1):36-43.

601 25. Lui S, Yao L, Xiao Y, Keedy SK, Reilly JL, Keefe RS, Tamminga CA, Keshavan

602 MS, Pearlson GD, Gong Q et al. Resting-state brain function in schizophrenia and

603 psychotic bipolar probands and their first-degree relatives. Psychological medicine.

$6042015 ; 45(1): 97-108$.

605 26. Liu H, Liu Z, Liang M, Hao Y, Tan L, Kuang F, Yi Y, Xu L, Jiang T. Decreased

606 regional homogeneity in schizophrenia: a resting state functional magnetic resonance

607 imaging study. Neuroreport. 2006; 17(1):19-22. 
608 27. Hoptman MJ, Zuo XN, Butler PD, Javitt DC, D'Angelo D, Mauro CJ, Milham MP.

609 Amplitude of low-frequency oscillations in schizophrenia: a resting state fMRI study.

610 Schizophrenia research. 2010; 117(1):13-20.

611 28. Qiu L, Fu X, Wang S, Tang Q, Chen X, Cheng L, Zhang F, Zhou Z, Tian L.

612 Abnormal regional spontaneous neuronal activity associated with symptom severity in

613 treatment-naive patients with obsessive-compulsive disorder revealed by resting-state

614 functional MRI. Neuroscience letters. 2017; 640:99-104.

615 29. Thorsen AL, Hagland P, Radua J, Mataix-Cols D, Kvale G, Hansen B, van den

616 Heuvel OA. Emotional Processing in Obsessive-Compulsive Disorder: A Systematic

617 Review and Meta-analysis of 25 Functional Neuroimaging Studies. Biological

618 psychiatry Cognitive neuroscience and neuroimaging. 2018; 3(6):563-571.

619 30. Eng GK, Sim K, Chen SH. Meta-analytic investigations of structural grey matter,

620 executive domain-related functional activations, and white matter diffusivity in

621 obsessive compulsive disorder: an integrative review. Neuroscience and biobehavioral

622 reviews. 2015; 52:233-257.

623 31. Butler PD, Silverstein SM, Dakin SC. Visual perception and its impairment in

624 schizophrenia. Biological psychiatry. 2008; 64(1):40-47. 
625 32. Javitt DC. When doors of perception close: bottom-up models of disrupted

626 cognition in schizophrenia. Annual review of clinical psychology. 2009; 5:249-275.

627 33. Lui S, Li T, Deng W, Jiang L, Wu Q, Tang H, Yue Q, Huang X, Chan RC, Collier

628 DA et al. Short-term effects of antipsychotic treatment on cerebral function in drug-

629 naive first-episode schizophrenia revealed by "resting state" functional magnetic

630 resonance imaging. Archives of general psychiatry. 2010; 67(8):783-792.

631 34. Xu Y, Zhuo C, Qin W, Zhu J, Yu C. Altered Spontaneous Brain Activity in

632 Schizophrenia: A Meta-Analysis and a Large-Sample Study. BioMed research

633 international. 2015; 2015:204628.

634 35. Turner JA, Chen H, Mathalon DH, Allen EA, Mayer AR, Abbott CC, Calhoun VD,

635 Bustillo J. Reliability of the amplitude of low-frequency fluctuations in resting state

636 fMRI in chronic schizophrenia. Psychiatry research. 2012; 201(3):253-255.

637 36. Turner JA, Damaraju E, van Erp TG, Mathalon DH, Ford JM, Voyvodic J, Mueller

638 BA, Belger A, Bustillo J, McEwen S et al. A multi-site resting state fMRI study on the

639 amplitude of low frequency fluctuations in schizophrenia. Frontiers in neuroscience.

$640 \quad 2013 ; 7: 137$. 
641 37. Hoexter MQ, Batistuzzo MC. Disentangling the Role of Amygdala Activation

642 in Obsessive-Compulsive Disorder. Biological psychiatry Cognitive neuroscience and

643 neuroimaging. 2018; 3(6):499-500.

644 38. Gao J, Zhou Y, Yang X, Luo J, Meng F, Zheng D, Li Z. Abnormalities within and

645 beyond the cortico-striato-thalamo-cortical circuitry in medication-free patients with

646 OCD revealed by the fractional amplitude of low-frequency fluctuations and resting-

647 state functional connectivity. Neuroscience letters. 2019; 712:134449.

648 39. Meng Z, Zhang Z, Fan Q, Li Y. Altered Fractional Amplitude of Low Frequency

649 Fluctuations in Unmedicated Female Patients with Obsessive-Compulsive Disorder.

650 Annual International Conference of the IEEE Engineering in Medicine and Biology

651 Society IEEE Engineering in Medicine and Biology Society Annual International

652 Conference. 2018; 2018:1144-1147.

653 40. Hare SM, Adhikari BM, Du X, Garcia L, Bruce H, Kochunov P, Simon JZ, Hong

654 LE. Local versus long-range connectivity patterns of auditory disturbance in

655 schizophrenia. Schizophrenia research. 2021; 228:262-270.

656 41. Yang XY, Sun J, Luo J, Zhong ZX, Li P, Yao SM, Xiong HF, Huang FF, Li ZJ.

657 Regional homogeneity of spontaneous brain activity in adult patients with obsessive- 
658 compulsive disorder before and after cognitive behavioural therapy. Journal of affective

659 disorders. 2015; 188:243-251.

660 42. Chen C, Wang HL, Wu SH, Huang H, Zou JL, Chen J, Jiang TZ, Zhou Y, Wang

661 GH. Abnormal Degree Centrality of Bilateral Putamen and Left Superior Frontal Gyrus

662 in Schizophrenia with Auditory Hallucinations: A Resting-state Functional Magnetic

663 Resonance Imaging Study. Chinese medical journal. 2015; 128(23):3178-3184.

664 43. Palaniyappan L, Liddle PF. Diagnostic discontinuity in psychosis: a combined

665 study of cortical gyrification and functional connectivity. Schizophrenia bulletin. 2014;

$66640(3): 675-684$.

667 44. Li P, Yang X, Greenshaw AJ, Li S, Luo J, Han H, Liu J, Zhong Z, Guo Z, Xiong H

668 et al. The effects of cognitive behavioral therapy on resting-state functional brain

669 network in drug-naive patients with obsessive-compulsive disorder. Brain and behavior.

$670 \quad 2018 ; 8(5): \mathrm{e} 00963$.

671 45. Luo L, Li Q, You W, Wang Y, Tang W, Li B, Yang Y, Sweeney JA, Li F, Gong Q.

672 Altered brain functional network dynamics in obsessive-compulsive disorder. Human

673 brain mapping. 2021.

674 46. Gross-Isseroff R, Hermesh H, Zohar J, Weizman A. Neuroimaging communality

675 between schizophrenia and obsessive compulsive disorder: a putative basis for schizo- 
676 obsessive disorder? The world journal of biological psychiatry : the official journal of

677 the World Federation of Societies of Biological Psychiatry. 2003; 4(3):129-134.

678 47. Goodman WK, Price LH, Rasmussen SA, Mazure C, Fleischmann RL, Hill CL,

679 Heninger GR, Charney DS. The Yale-Brown Obsessive Compulsive Scale. I.

680 Development, use, and reliability. Archives of general psychiatry. 1989; 46(11):1006-

$681 \quad 1011$.

682 48. Hamilton M. The assessment of anxiety states by rating. The British journal of

683 medical psychology. 1959; 32(1):50-55.

684 49. Hamilton M. Development of a rating scale for primary depressive illness. The

685 British journal of social and clinical psychology. 1967; 6(4):278-296.

686 50. Oldfield RC. The assessment and analysis of handedness: the Edinburgh inventory.

687 Neuropsychologia. 1971; 9(1):97-113.

688 51. Yan CG, Wang XD, Zuo XN, Zang YF. DPABI: Data Processing \& Analysis for

689 (Resting-State) Brain Imaging. Neuroinformatics. 2016; 14(3):339-351.

690 52. Power JD, Barnes KA, Snyder AZ, Schlaggar BL, Petersen SE. Spurious but

691 systematic correlations in functional connectivity MRI networks arise from subject

692 motion. NeuroImage. 2012; 59(3):2142-2154. 
693 53. Yan CG, Cheung B, Kelly C, Colcombe S, Craddock RC, Di Martino A, Li Q, Zuo

694 XN, Castellanos FX, Milham MP. A comprehensive assessment of regional variation in

695 the impact of head micromovements on functional connectomics. NeuroImage. 2013;

$69676: 183-201$.

697 54. Friston KJ, Williams S, Howard R, Frackowiak RS, Turner R. Movement-related

698 effects in fMRI time-series. Magnetic resonance in medicine. 1996; 35(3):346-355.

699 55. Chao-Gan Y, Yu-Feng Z. DPARSF: A MATLAB Toolbox for "Pipeline" Data

700 Analysis of Resting-State fMRI. Frontiers in systems neuroscience. 2010; 4:13.

701 56. Lv Y, Li L, Song Y, Han Y, Zhou C, Zhou D, Zhang F, Xue Q, Liu J, Zhao L et al.

702 The Local Brain Abnormalities in Patients With Transient Ischemic Attack: A Resting-

703 State fMRI Study. Frontiers in neuroscience. 2019; 13:24.

704 57. Zhao N, Yuan LX, Jia XZ, Zhou XF, Deng XP, He HJ, Zhong J, Wang J, Zang YF.

705 Intra- and Inter-Scanner Reliability of Voxel-Wise Whole-Brain Analytic Metrics for

706 Resting State fMRI. Frontiers in neuroinformatics. 2018; 12:54.

707 58. Beucke JC, Sepulcre J, Talukdar T, Linnman C, Zschenderlein K, Endrass T,

708 Kaufmann C, Kathmann N. Abnormally high degree connectivity of the orbitofrontal

709 cortex in obsessive-compulsive disorder. JAMA psychiatry. 2013; 70(6):619-629. 
711 et al. Abnormal Degree Centrality Associated With Cognitive Dysfunctions in Early

712 Bipolar Disorder. Frontiers in psychiatry. 2019; 10:140.

713 60. Zhang J, Su J, Wang M, Zhao Y, Zhang QT, Yao Q, Lu H, Zhang H, Li GF, Wu YL

714 et al. The sensorimotor network dysfunction in migraineurs without aura: a resting-state

715 fMRI study. Journal of neurology. 2017; 264(4):654-663.

716 61. Wang JB, Zheng LJ, Cao QJ, Wang YF, Sun L, Zang YF, Zhang H. Inconsistency

717 in Abnormal Brain Activity across Cohorts of ADHD-200 in Children with Attention

718 Deficit Hyperactivity Disorder. Frontiers in neuroscience. 2017; 11:320.

719 62. Ping L, Su-Fang L, Hai-Ying H, Zhang-Ye D, Jia L, Zhi-Hua G, Hong-Fang X, Yu-

720 Feng Z, Zhan-Jiang L. Abnormal Spontaneous Neural Activity in Obsessive-

721 Compulsive Disorder: A Resting-State Functional Magnetic Resonance Imaging Study.

722 PloS one. 2013; 8(6):e67262.

723 63. Stern ER, Fitzgerald KD, Welsh RC, Abelson JL, Taylor SF. Resting-state

724 functional connectivity between fronto-parietal and default mode networks in 725 obsessive-compulsive disorder. PloS one. 2012; 7(5):e36356. 
727 Abnormal small-world architecture of top-down control networks in obsessive-

728 compulsive disorder. Journal of psychiatry \& neuroscience : JPN. 2011; 36(1):23-31.

729 65. Boedhoe PSW, Schmaal L, Abe Y, Alonso P, Ameis SH, Anticevic A, Arnold PD,

730 Batistuzzo MC, Benedetti F, Beucke JC et al. Cortical Abnormalities Associated With

731 Pediatric and Adult Obsessive-Compulsive Disorder: Findings From the ENIGMA

732 Obsessive-Compulsive Disorder Working Group. The American journal of psychiatry.

$7332018 ; 175(5): 453-462$.

734 66. Torrey EF. Schizophrenia and the inferior parietal lobule. Schizophrenia research.

735 2007; 97(1-3):215-225.

736 67. Yan CG, Craddock RC, Zuo XN, Zang YF, Milham MP. Standardizing the intrinsic

737 brain: towards robust measurement of inter-individual variation in 1000 functional

738 connectomes. NeuroImage. 2013; 80:246-262.

739 68. Cabeza R, Nyberg L. Neural bases of learning and memory: functional

740 neuroimaging evidence. Current opinion in neurology. 2000; 13(4):415-421.

741 69. Niu Q, Yang L, Song X, Chu C, Liu H, Zhang L, Li Y, Zhang X, Cheng J, Li Y.

742 Abnormal resting-state brain activities in patients with first-episode obsessive-

743 compulsive disorder. Neuropsychiatric disease and treatment. 2017; 13:507-513. 
744 70. Nierenberg J, Salisbury DF, Levitt JJ, David EA, McCarley RW, Shenton ME.

745 Reduced left angular gyrus volume in first-episode schizophrenia. The American

746 journal of psychiatry. 2005; 162(8):1539-1541.

747 71. Onitsuka T, McCarley RW, Kuroki N, Dickey CC, Kubicki M, Demeo SS, Frumin

748 M, Kikinis R, Jolesz FA, Shenton ME. Occipital lobe gray matter volume in male

749 patients with chronic schizophrenia: A quantitative MRI study. Schizophrenia research.

$750 \quad 2007 ; 92(1-3): 197-206$.

751 72. Fan J, Zhong M, Gan J, Liu W, Niu C, Liao H, Zhang H, Tan C, Yi J, Zhu X.

752 Spontaneous neural activity in the right superior temporal gyrus and left middle

753 temporal gyrus is associated with insight level in obsessive-compulsive disorder.

754 Journal of affective disorders. 2017; 207:203-211.

755 73. Piras F, Piras F, Chiapponi C, Girardi P, Caltagirone C, Spalletta G. Widespread

756 structural brain changes in OCD: a systematic review of voxel-based morphometry

757 studies. Cortex; a journal devoted to the study of the nervous system and behavior. 2015;

$758 \quad 62: 89-108$.

759 74. Yu R, Hsieh MH, Wang HL, Liu CM, Liu CC, Hwang TJ, Chien YL, Hwu HG,

760 Tseng WY. Frequency dependent alterations in regional homogeneity of baseline brain

761 activity in schizophrenia. PloS one. 2013; 8(3):e57516. 
762 75. Farber RH, Clementz BA, Swerdlow NR. Characteristics of open- and closed-loop

763 smooth pursuit responses among obsessive-compulsive disorder, schizophrenia, and

764 nonpsychiatric individuals. Psychophysiology. 1997; 34(2):157-162.

765 76. Szeszko PR, Ardekani BA, Ashtari M, Malhotra AK, Robinson DG, Bilder RM,

766 Lim KO. White matter abnormalities in obsessive-compulsive disorder: a diffusion

767 tensor imaging study. Archives of general psychiatry. 2005; 62(7):782-790.

768 77. Gao X, Zhang W, Yao L, Xiao Y, Liu L, Liu J, Li S, Tao B, Shah C, Gong Q et al.

769 Association between structural and functional brain alterations in drug-free patients

770 with schizophrenia: a multimodal meta-analysis. Journal of psychiatry \& neuroscience :

771 JPN. 2018; 43(2):131-142.

772 78. Moreira PS, Marques P, Soriano-Mas C, Magalhães R, Sousa N, Soares JM,

773 Morgado P. The neural correlates of obsessive-compulsive disorder: a multimodal

774 perspective. Translational psychiatry. 2017; 7(8):e1224.

775 79. Gonçalves OF, Marques TR, Lori NF, Sampaio A, Branco MC. Obsessive-

776 compulsive disorder as a visual processing impairment. Medical hypotheses. 2010;

777 74(1):107-109. 
778 80. Revheim N, Butler PD, Schechter I, Jalbrzikowski M, Silipo G, Javitt DC. Reading

779 impairment and visual processing deficits in schizophrenia. Schizophrenia research.

$780 \quad 2006 ; 87(1-3): 238-245$.

781 81. Pratt H, Bleich N, Mittelman N. Spatio-temporal distribution of brain activity

782 associated with audio-visually congruent and incongruent speech and the McGurk

783 Effect. Brain and behavior. 2015; 5(11):e00407.

784 82. Luthra S, Correia JM, Kleinschmidt DF, Mesite L, Myers EB. Lexical Information

785 Guides Retuning of Neural Patterns in Perceptual Learning for Speech. Journal of

786 cognitive neuroscience. 2020; 32(10):2001-2012.

787 83. Branzi FM, Pobric G, Jung J, Lambon Ralph MA. The Left Angular Gyrus Is

788 Causally Involved in Context-dependent Integration and Associative Encoding during

789 Narrative Reading. Journal of cognitive neuroscience. 2021:1-14.

790 84. Scheinin A, Kantonen O, Alkire M, Långsjö J, Kallionpää RE, Kaisti K, Radek L,

791 Johansson J, Sandman N, Nyman M et al. Foundations of Human Consciousness:

792 Imaging the Twilight Zone. The Journal of neuroscience : the official journal of the

793 Society for Neuroscience. 2021; 41(8):1769-1778. 
794 85. Schilbach L, Eickhoff SB, Rotarska-Jagiela A, Fink GR, Vogeley K. Minds at rest?

795 Social cognition as the default mode of cognizing and its putative relationship to the

796 "default system" of the brain. Consciousness and cognition. 2008; 17(2):457-467.

797 86. Fan J, Gan J, Liu W, Zhong M, Liao H, Zhang H, Yi J, Chan RCK, Tan C, Zhu X.

798 Resting-State Default Mode Network Related Functional Connectivity Is Associated

799 With Sustained Attention Deficits in Schizophrenia and Obsessive-Compulsive

800 Disorder. Frontiers in behavioral neuroscience. 2018; 12:319.

801 87. Qiu L, Tian L, Pan C, Zhu R, Liu Q, Yan J, Zhao Q, Yuan H, Han Y, Yue W et al.

802 Neuroanatomical circuitry associated with exploratory eye movement in schizophrenia:

803 a voxel-based morphometric study. PloS one. 2011; 6(10):e25805.

804 88. Matsue Y, Okuma T, Saito H, Aneha S, Ueno T, Chiba H, Matsuoka H. Saccadic

805 eye movements in tracking, fixation, and rest in schizophrenic and normal subjects.

806 Biological psychiatry. 1986; 21(4):382-389.

807 89. Matsushima E, Kojima T, Ohta K, Obayashi S, Nakajima K, Kakuma T, Ando H,

808 Ando K, Toru M. Exploratory eye movement dysfunctions in patients with

809 schizophrenia: possibility as a discriminator for schizophrenia. Journal of psychiatric

810 research. 1998; 32(5):289-295. 
811 90. Suzuki M, Takahashi S, Matsushima E, Tsunoda M, Kurachi M, Okada T, Hayashi

812 T, Ishii Y, Morita K, Maeda H et al. Exploratory eye movement dysfunction as a

813 discriminator for schizophrenia : a large sample study using a newly developed digital

814 computerized system. European archives of psychiatry and clinical neuroscience. 2009;

815 259(3):186-194.

816 91. Qiu L, Yan H, Zhu R, Yan J, Yuan H, Han Y, Yue W, Tian L, Zhang D. Correlations

817 between exploratory eye movement, hallucination, and cortical gray matter volume in

818 people with schizophrenia. BMC psychiatry. 2018; 18(1):226.

819 92. Xia J, Fan J, Du H, Liu W, Li S, Zhu J, Yi J, Tan C, Zhu X. Abnormal spontaneous

820 neural activity in the medial prefrontal cortex and right superior temporal gyrus

821 correlates with anhedonia severity in obsessive-compulsive disorder. Journal of

822 affective disorders. 2019; 259:47-55.

823 93. Jaspers-Fayer F, Lin SY, Chan E, Ellwyn R, Lim R, Best J, Belschner L, Lang D,

824 Heran MKM, Woodward TS et al. Neural correlates of symptom provocation in

825 pediatric obsessive-compulsive disorder. NeuroImage Clinical. 2019; 24:102034. 
828 Demographic, clinical and head-motion characteristics of the samples in this study.

\begin{tabular}{|c|c|c|c|c|c|}
\hline Variables & HC $(n=60)$ & $\operatorname{OCD}(n=27)$ & $S Z(n=22)$ & $\begin{array}{c}\text { Statistics (F/ } \\
\chi^{2 / T / Z / H)}\end{array}$ & $\begin{array}{c}P \\
\text { value }\end{array}$ \\
\hline Age (years) & $32.87 \pm 10.78^{a}$ & $26.89 \pm 8.15$ & $33.41 \pm 11.03$ & 3.65 & $0.029^{*}$ \\
\hline Education(years) & $14.02 \pm 3.72$ & $13.26 \pm 2.96$ & $10.77 \pm 4.74$ & 5.94 & $0.004^{* c}$ \\
\hline $\operatorname{Sex}(M / F)$ & $38 / 22$ & $21 / 6$ & $11 / 11$ & 4.12 & $0.128^{\#}$ \\
\hline Disease duration(years) & - & $3.00(1.00,6.00)^{\mathrm{b}}$ & $1.29(0.17,3.25)$ & -2.61 & $0.009^{\&}$ \\
\hline PANSS positive score & - & - & $27.18 \pm 4.63$ & - & - \\
\hline PANSS negative score & - & - & $19.82 \pm 5.14$ & - & - \\
\hline PANSS general score & - & - & $46.50 \pm 7.58$ & - & - \\
\hline PANSS total score & - & - & $93.50 \pm 12.39$ & - & - \\
\hline \multicolumn{6}{|l|}{ Y-BOCS score } \\
\hline Obsession score & - & $12.33 \pm 3.87$ & - & - & - \\
\hline Compulsive score & - & $8.70 \pm 2.95$ & - & - & - \\
\hline Total score & - & $21.04 \pm 5.93$ & - & - & - \\
\hline HARS score & - & $14.00(12.00,19.00)$ & - & - & - \\
\hline 24-HDRS score & - & $16.30 \pm 7.83$ & - & - & - \\
\hline Mean FD & $0.07(0.06,0.12)$ & $0.08(0.05,0.12)$ & $0.07(0.05,0.10)$ & 0.95 & $0.623^{\nabla}$ \\
\hline
\end{tabular}

829 Note: ${ }^{\text {a }}$ Values are presented as mean \pm SD.

$830{ }^{\mathrm{b}}$ Values are presented as median (first quartile, third quartile).

831 *, one-way ANOVA ; ${ }^{*}, \mathrm{x}^{2}$ test; ${ }^{\star}$, Mann-Whitney U test; ${ }^{\nabla}$,Kruskal-Wallis test.

$832{ }^{c}$ Post-hoc analysis showed that Patients with OCD differed significantly from controls

833 (Bonferroni, $P<0.05$ ).

$834{ }^{\mathrm{d}}$ Post-hoc analysis showed that Patients with SZ differed significantly from controls

835 (Bonferroni, $P<0.05$ ).

$836 P<0.05$ is considered significant.

837 Abbreviations: HC, healthy controls; OCD, patients with obsessive-compulsive

838 disorder; SZ, patients with schizophrenia; PANSS, Positive and Negative Syndrome

839 Scale; HARS, the Hamilton Anxiety Rating Scale; 24-HDRS, the 24-item Hamilton

840 Rating Scale for Depression; Y-BOCS, the Yale-Brown Obsessive-Compulsive Scale;

841 Mean FD, mean framewise displacement.

842 
845 The ALFF, ReHo and DC clusters with significant between-group differences (Cluster-level $P_{\mathrm{FWE}}$

\begin{tabular}{|c|c|c|c|c|c|c|c|c|c|}
\hline \multirow{2}{*}{ Feature } & \multirow{2}{*}{ Index } & \multirow{2}{*}{$\begin{array}{c}\text { Cluster } \\
\text { size }\end{array}$} & \multirow{2}{*}{ Brain regions } & \multirow{2}{*}{ side } & \multirow{2}{*}{ BA } & \multicolumn{3}{|c|}{ MNI coordinate } & \multirow{2}{*}{ Peak F } \\
\hline & & & & & & $\mathbf{x}$ & $\mathbf{y}$ & $\mathbf{z}$ & \\
\hline \multirow[t]{9}{*}{ ALFF } & 1 & 47 & Angular gyrus & $\mathrm{R}$ & 39 & 42 & -51 & 30 & 29.20 \\
\hline & 2 & 40 & Superior temporal gyrus & $\mathrm{L}$ & 48 & -45 & -36 & 24 & 8.77 \\
\hline & & & Insula & $\mathrm{L}$ & 48 & -36 & -18 & 15 & 11.20 \\
\hline & & & Rolandic operculum & $\mathrm{L}$ & 48 & -36 & -27 & 18 & 10.85 \\
\hline & 3 & 37 & Middle frontal gyrus & $\mathrm{L}$ & 9 & -36 & 9 & 51 & 21.84 \\
\hline & & & Precentral gyrus & $\mathrm{L}$ & 6 & -36 & 3 & 42 & 12.08 \\
\hline & 4 & 37 & Postcentral gyrus & $\mathrm{L}$ & 4 & -24 & -30 & 66 & 11.39 \\
\hline & 5 & 33 & Supramarginal gyrus & $\mathrm{R}$ & 2 & 45 & -33 & 39 & 13.06 \\
\hline & & & Inferior parietal lobule & $\mathrm{R}$ & 2 & 48 & -36 & 51 & 11.96 \\
\hline \multirow[t]{2}{*}{ ReHo } & 1 & 105 & Angular gyrus & $\mathrm{R}$ & 39 & 42 & -51 & 30 & 40.14 \\
\hline & & & Middle occipital gyrus & $\mathrm{R}$ & 19 & 39 & -72 & 33 & 18.76 \\
\hline \multirow[t]{4}{*}{$\mathrm{DC}$} & 1 & 105 & Lingual gyrus & $\mathrm{R}$ & 18 & 12 & -87 & -12 & 17.18 \\
\hline & & & $\begin{array}{l}\text { Calcarine fissure and } \\
\text { surrounding cortex }\end{array}$ & $\mathrm{R}$ & 17 & 6 & -78 & 3 & 8.32 \\
\hline & 2 & 49 & Inferior parietal lobule & $\mathrm{R}$ & 40 & 54 & -57 & 48 & 14.42 \\
\hline & & & Angular gyrus & $\mathrm{R}$ & 39 & 60 & -57 & 30 & 10.19 \\
\hline
\end{tabular}

$846<0.05$ when voxel-level threshold was $P<0.001)$.

847 Abbreviations: BA, Brodmann area; MNI, Montreal Neurological Institute; R, right; L, left; ALFF,

848 amplitude of low-frequency fluctuation; ReHo, regional homogeneity; DC, degree centrality. 


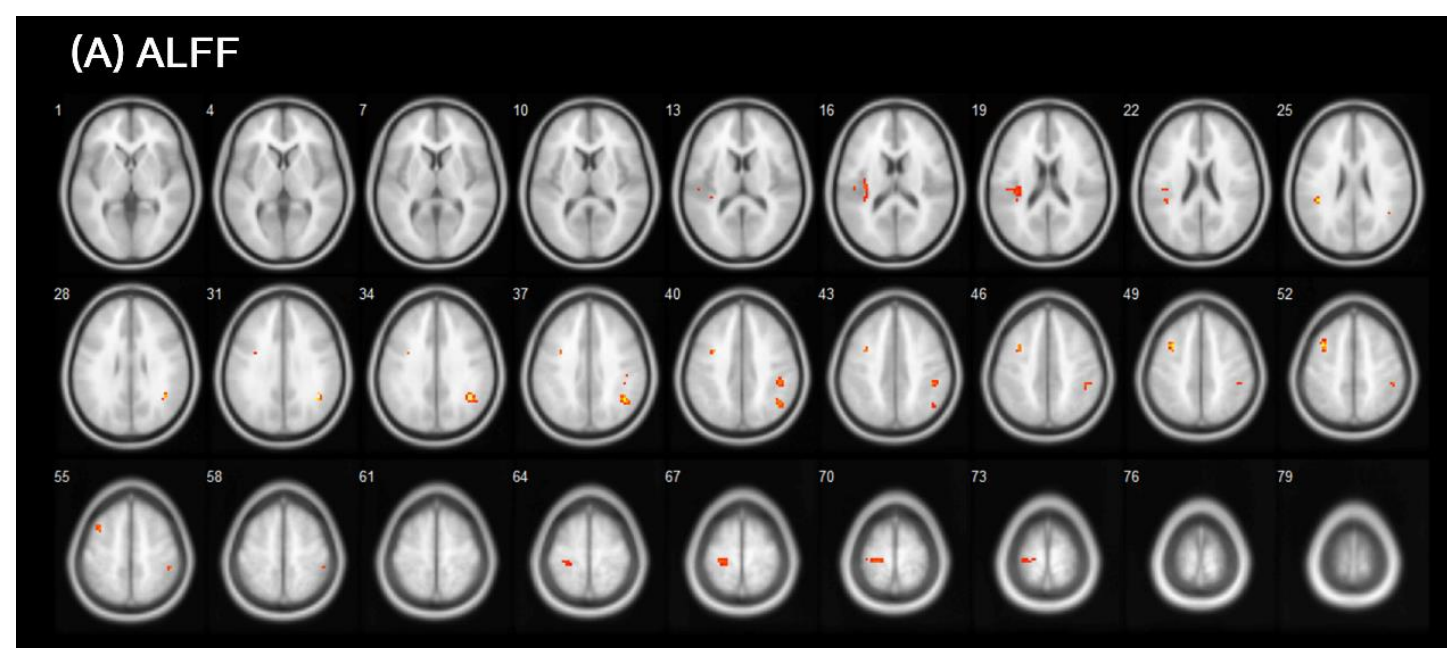

\section{(B) ReHo}

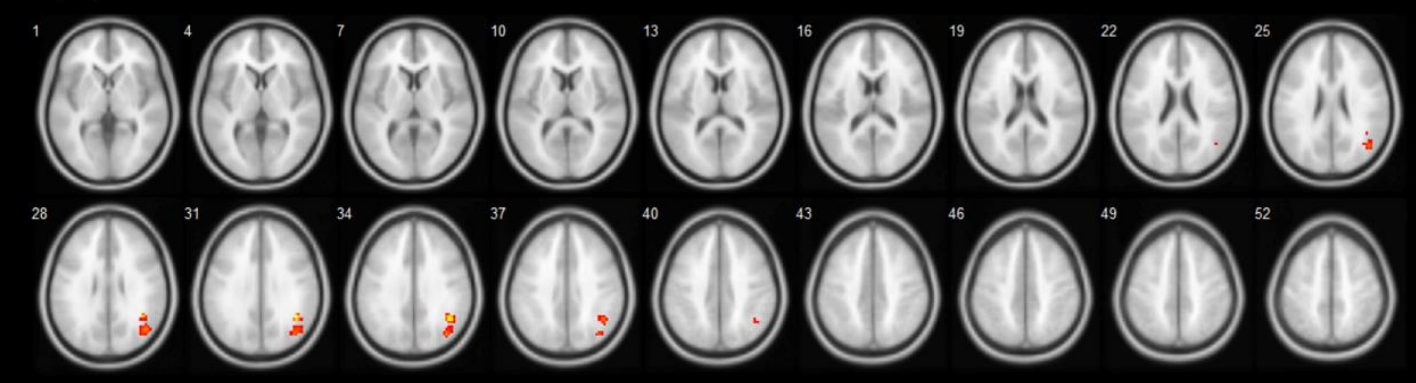

(C) DC
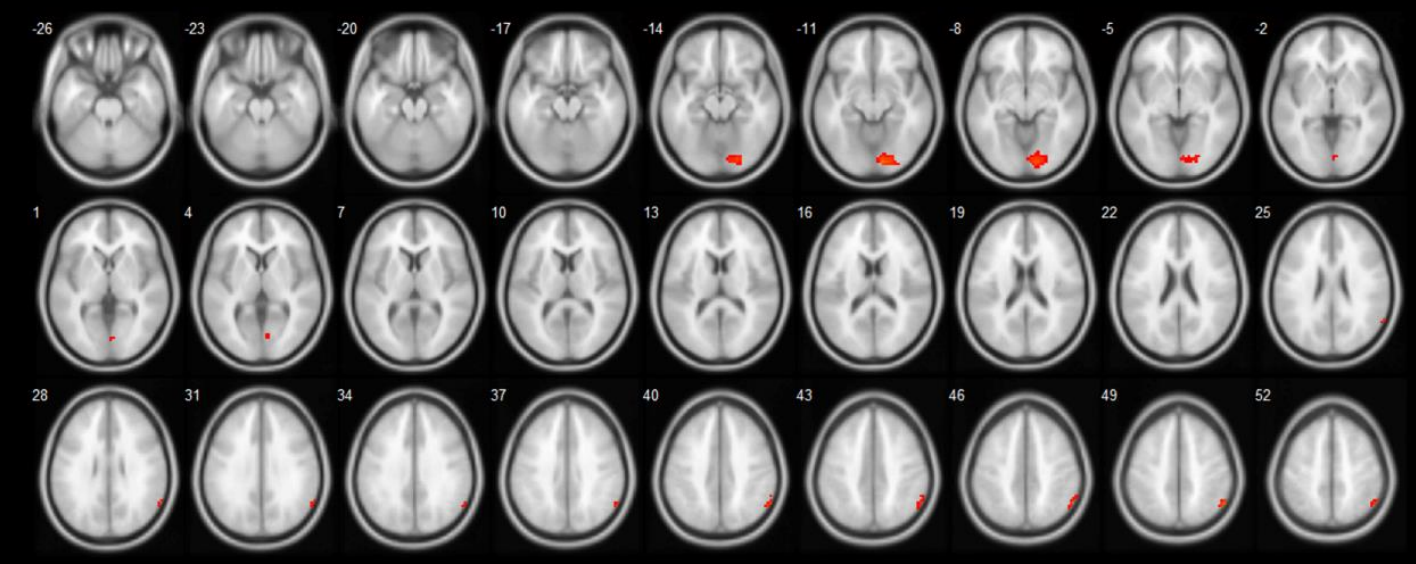

$$
\text { F value }
$$

\section{$851 \quad$ Figure 1}

852 Significant differences in ALFF, ReHo and DC among healthy controls, patients with schizophrenia

853 and obsessive-compulsive disorder. 
854 (A). Brain regions with significant differences (cluster-level $P_{\mathrm{FWE}}<0.05$ when the voxel-level 855 threshold was $P<0.001$ ) of the amplitude of low-frequency fluctuation among healthy controls, 856 patients with schizophrenia and obsessive-compulsive disorder.

857 (B). Brain regions with significant differences (cluster-level $P_{\mathrm{FWE}}<0.05$ when the voxel-level 858 threshold was $P<0.001)$ of the regional homogeneity among healthy controls, patients with 859 schizophrenia and obsessive-compulsive disorder.

860 (C). Brain regions with significant differences (cluster-level $P_{\text {FWE }}<0.05$ when the voxel-level 861 threshold was $P<0.001)$ of the degree of centrality among healthy controls, patients with 862 schizophrenia and obsessive-compulsive disorder.

863 Notes: The colored bars show F values.

864 Abbreviations: L, left; R, right; ALFF, amplitude of low-frequency fluctuation; ReHo, regional 865 homogeneity; DC, degree centrality 
(A)
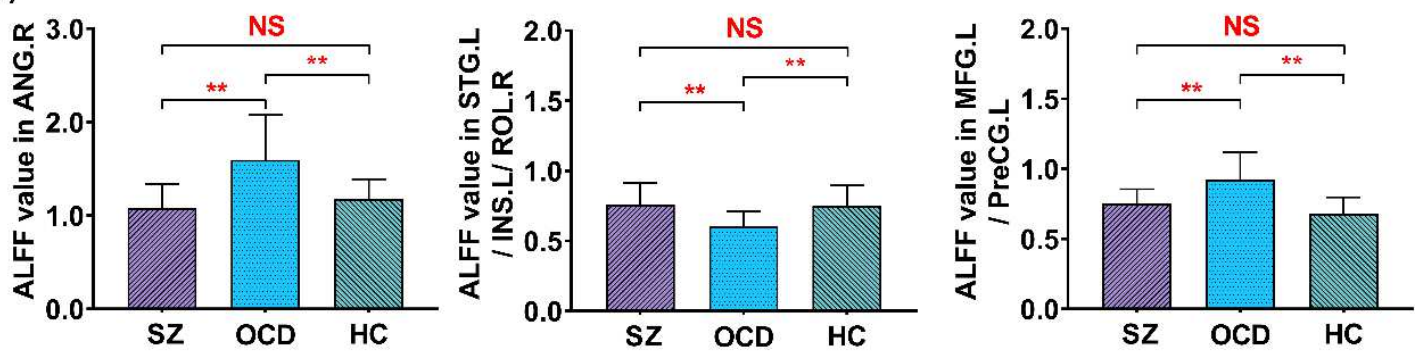

(B)
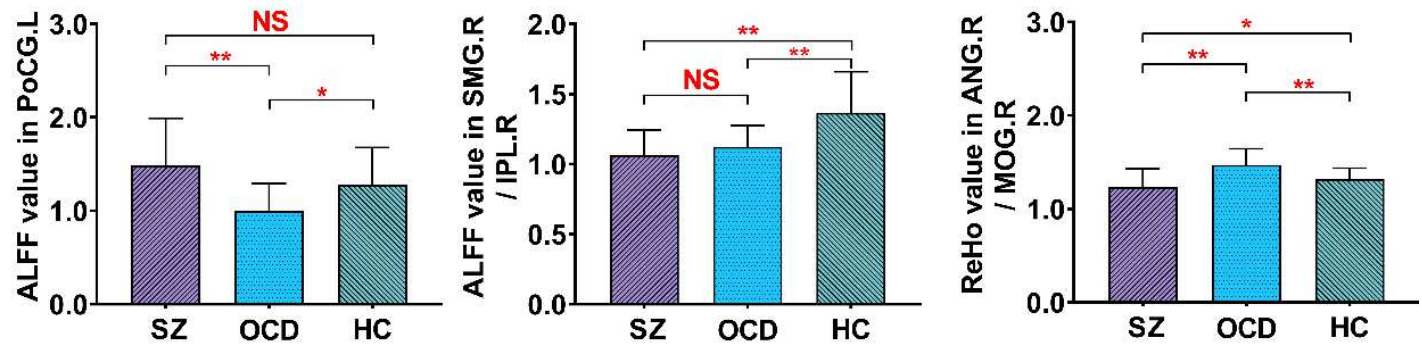

(C)
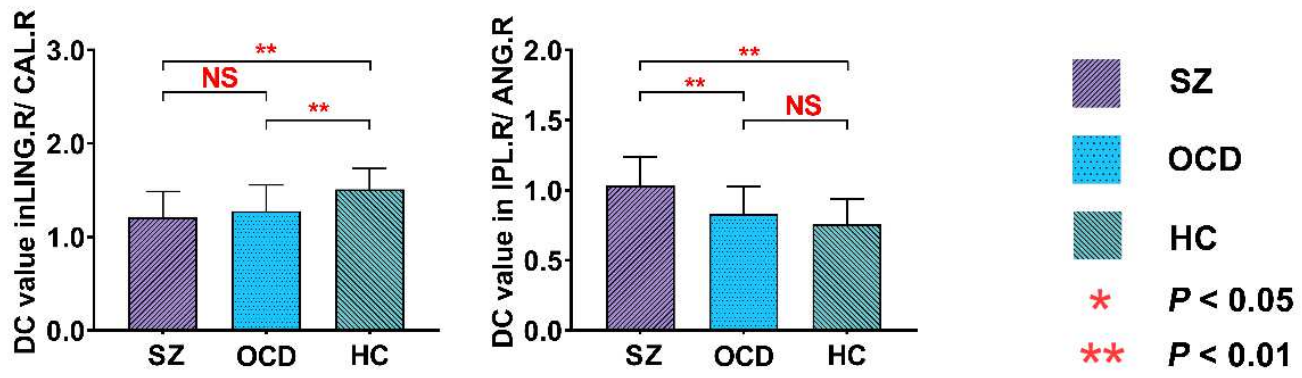

Figure 2

870 Histogram plots illustrate the mean ALFF/ReHo/DC values of the clusters showing significant

871 differences among the healthy controls, patients with schizophrenia and obsessive-compulsive

872 disorder.

873 (A). The mean ALFF values in the ANG.R, STG.L/INS.L/ROL.R, MFG.L/PreCG.L, PoCG.L, and

874 SMG.R/IPL.R among the three groups.

875 (B). The mean ReHo values in the ANG.R/MoG.R among the three groups.

876 (C). The mean DC values in the ING.R/CAL.R and IPL.R/ANG.R.

877 Error bars reflect the SD.

878 Abbreviations: HC, healthy controls; OCD, patients with obsessive-compulsive disorder; SZ,

879 patient with schizophrenia; ALFF, amplitude of low-frequency fluctuation; ReHo, regional

880 homogeneity; DC, degree centrality; ANG.R, right angular gyrus; STG.L/INS.L/ROL.R, left

881 superior temporal gyrus/insula/rolandic operculum; MFG.L/PreCG.L, left middle frontal

882 gyrus/precentral gyrus; PoCG.L, left postcentral gyrus, SMG.R/IPL.R, right supramarginal

883 gyrus/inferior parietal lobule; ANG.R/MoG.R, right angular gyrus/middle occipital gyrus; 
884 ING.R/CAL.R, right lingual gyrus/ calcarine fissure and surrounding cortex; IPL.R/ANG.R, right 885 inferior parietal lobule/ angular gyrus.

886 
(A)
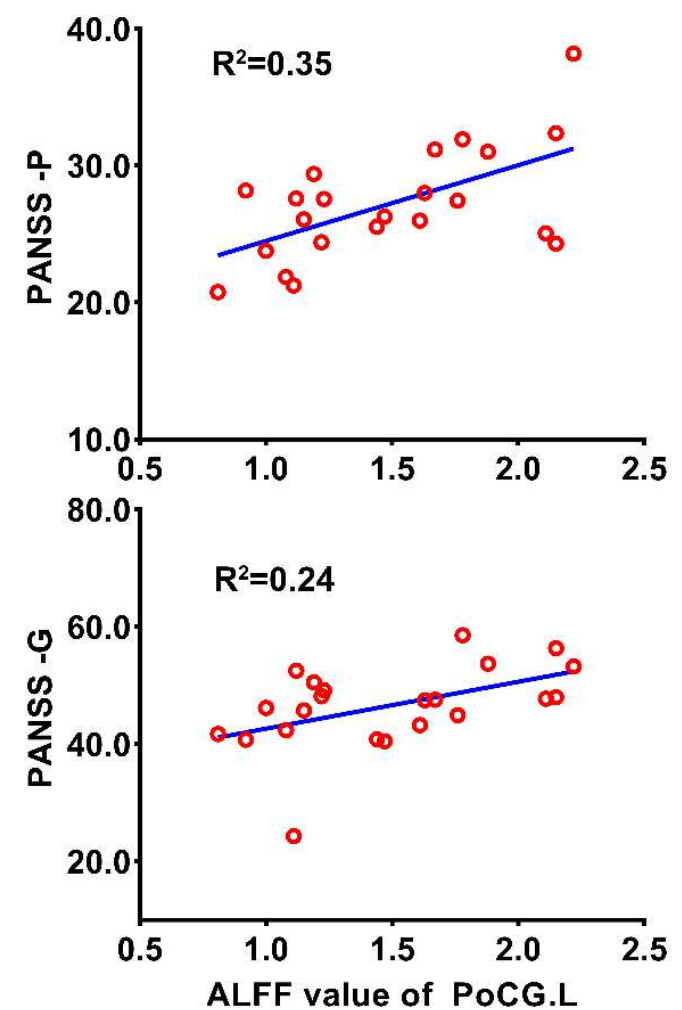

(B)
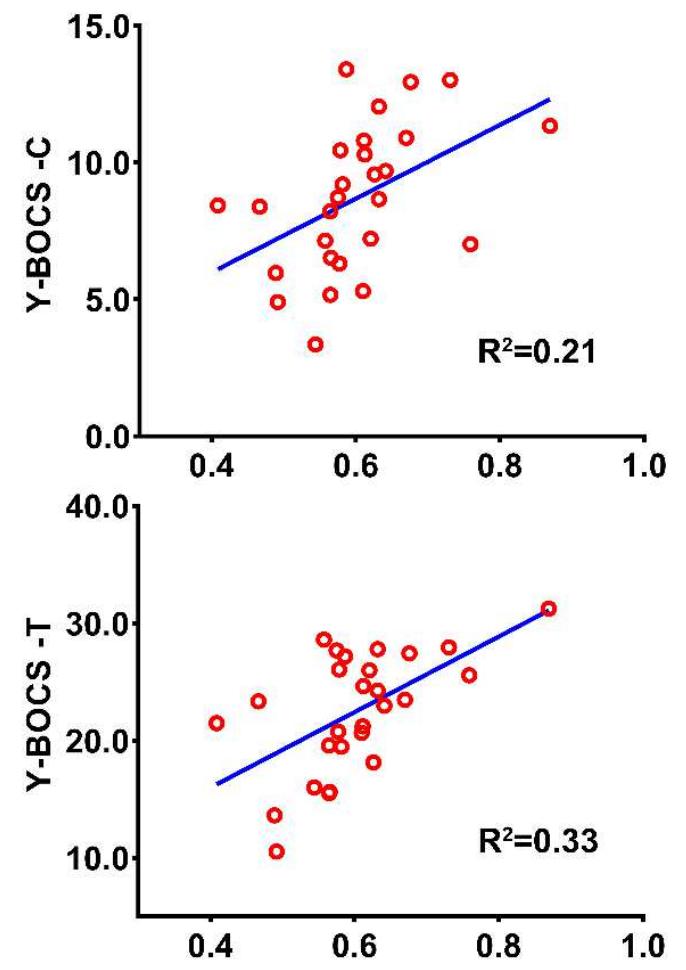

ALFF value of STG.L/INS.L/ROL.R

Figure 3:

889 Scatter plots show the relationships between the ALFF values and the clinical symptoms in patients

890 with schizophrenia and obsessive-compulsive disorder.

891 (A). The ALFF values of the left postcentral gyrus of patients with SZ were positively correlated

892 with the scores of PANSS general pathology scale and positive subscale; (B). The ALFF values in

893 the left superior temporal gyrus/insula/rolandic operculum brain area of patients with OCD were

894 positively correlated with compulsion subscale scores and the total scores of Y-BOCS.

895 Abbreviations: PANSS, Positive and Negative Syndrome Scale; Y-BOCS, the Yale-Brown

896 Obsessive-Compulsive Scale; ALFF, amplitude of low-frequency fluctuation; PANSS-P, PANSS

897 positive score; PANSS-G, PANSS general psychopathological score; Y-BOCS -C, Y-BOCS

898 compulsive score; YBOCS-T, Y-BOCS total score; PoCG.L, left postcentral gyrus;

899 STG.L/INS.L/ROL.R, left superior temporal gyrus/insula/rolandic operculum; $\mathrm{R}^{2}$, the coefficient of

900 determination.

901

902 


\section{Figures}

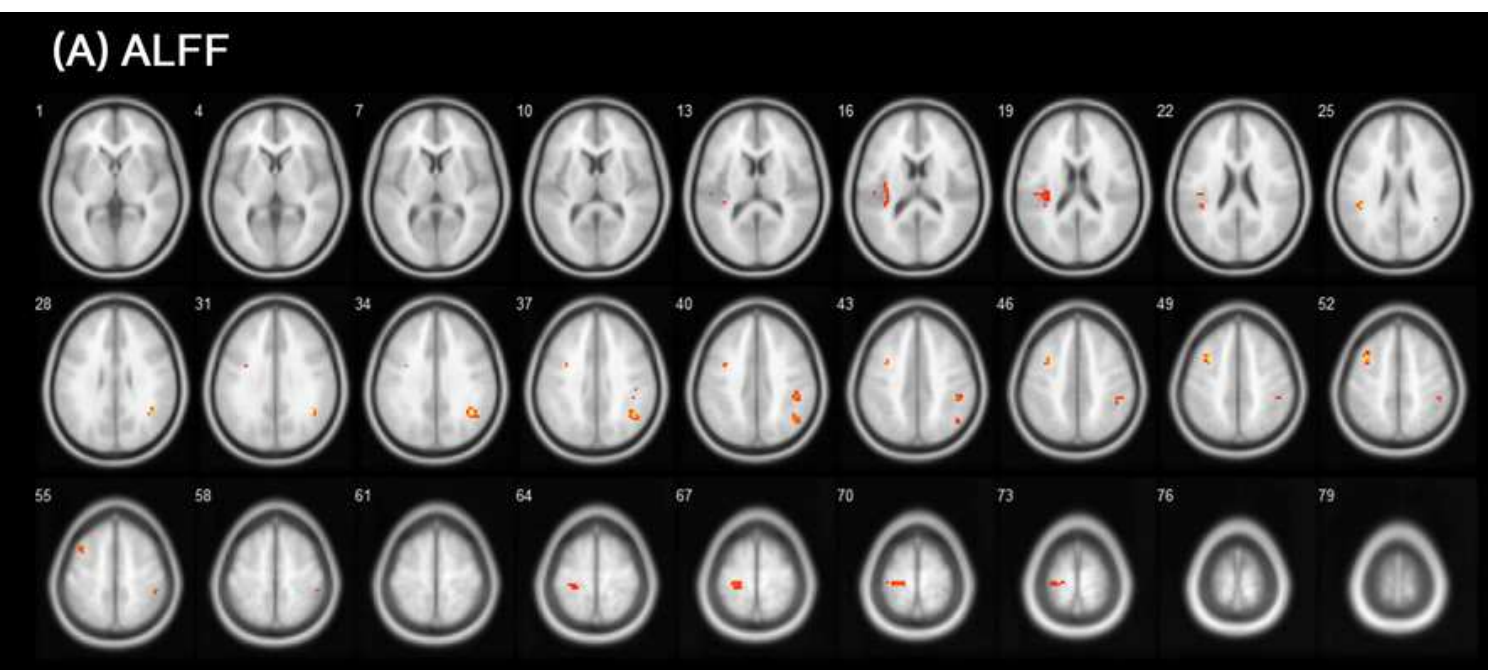

(B) $\mathrm{ReHo}$

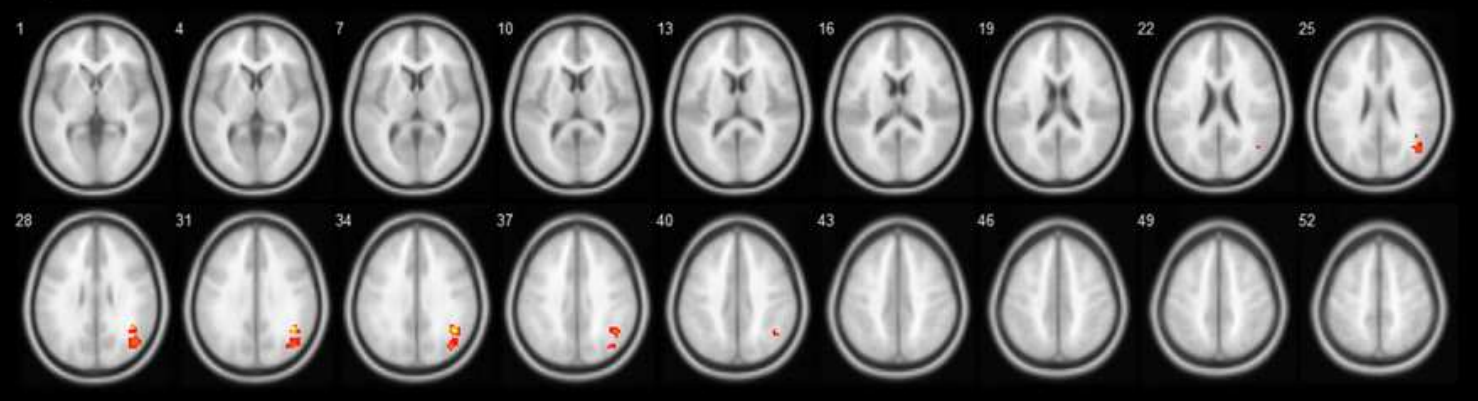

(C) DC

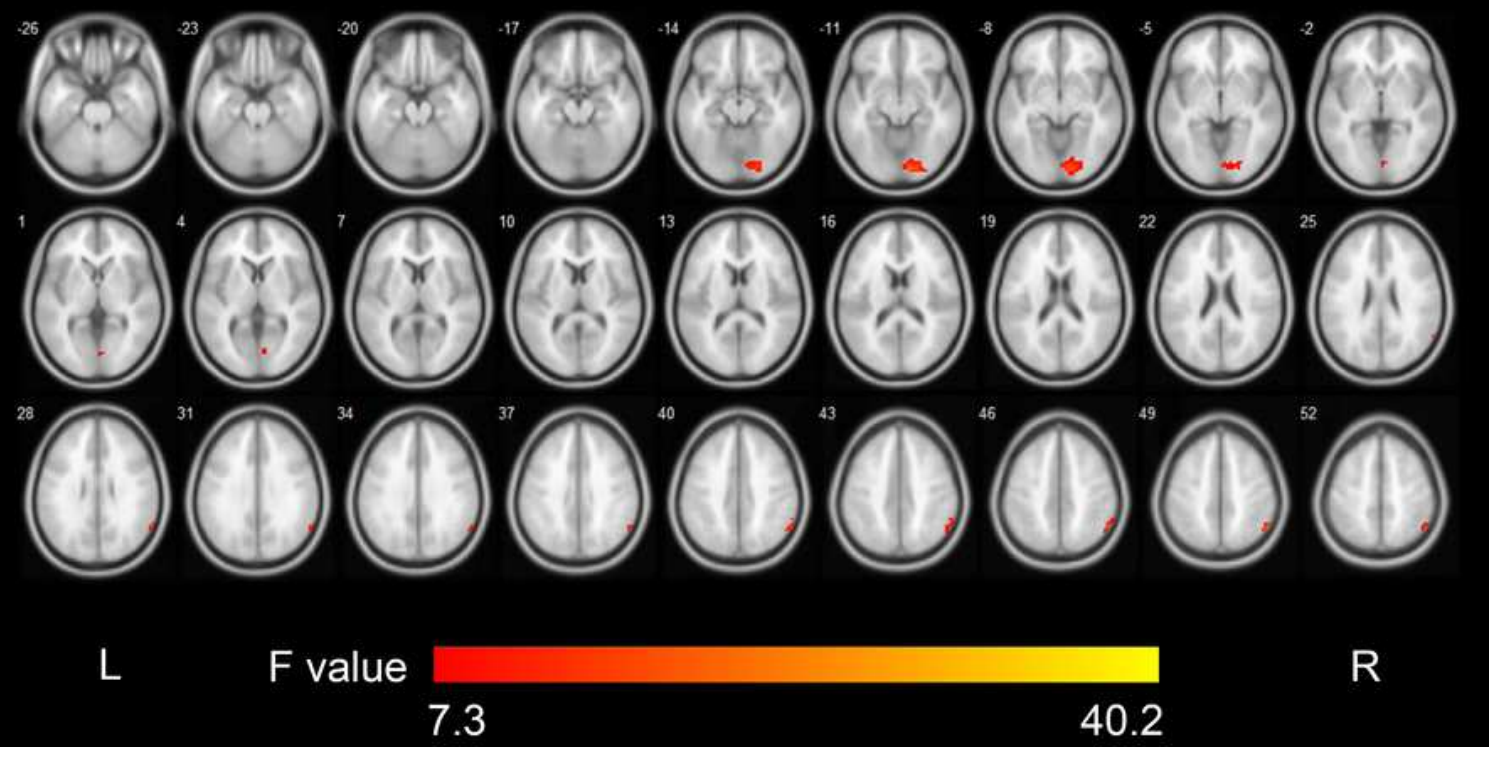

Figure 1

Significant differences in ALFF, ReHo and DC among healthy controls, patients with schizophrenia and obsessive-compulsive disorder. (A) Brain regions with significant differences (cluster-level PFWE $<0.05$ when the voxel-level threshold was $\mathrm{P}<0.001$ ) of the amplitude of low-frequency fluctuation among 
healthy controls, patients with schizophrenia and obsessive-compulsive disorder. (B). Brain regions with significant differences (cluster-level PFWE $<0.05$ when the voxel-level threshold was $P<0.001$ ) of the regional homogeneity among healthy controls, patients with schizophrenia and obsessive-compulsive disorder. (C). Brain regions with significant differences (cluster-level PFWE $<0.05$ when the voxel-level threshold was $P<0.001$ ) of the degree of centrality among healthy controls, patients with schizophrenia and obsessive-compulsive disorder. Notes: The colored bars show F values. Abbreviations: L, left; R, right; ALFF, amplitude of low-frequency fluctuation; ReHo, regional homogeneity; DC, degree centrality

(A)
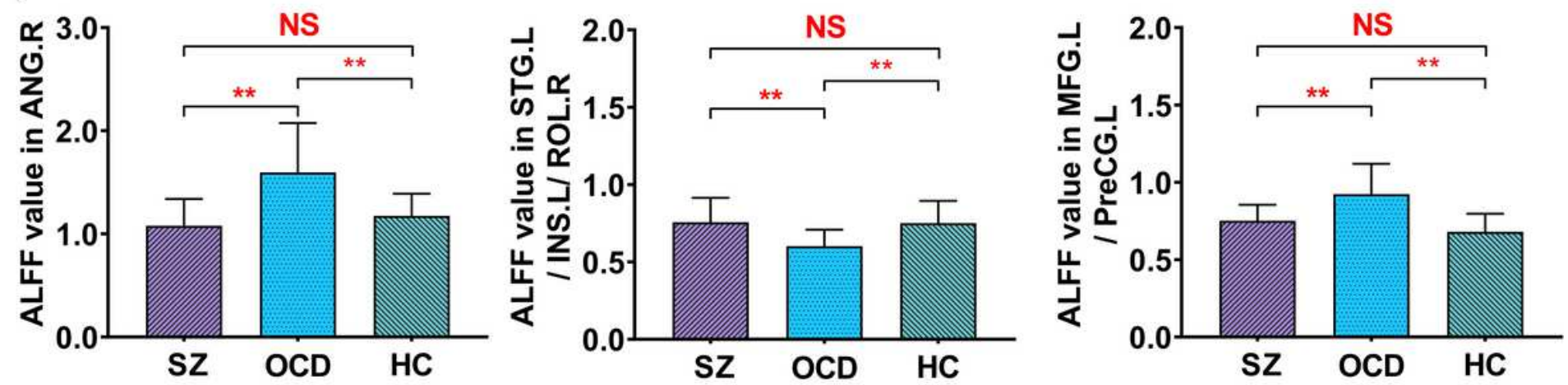

(B)
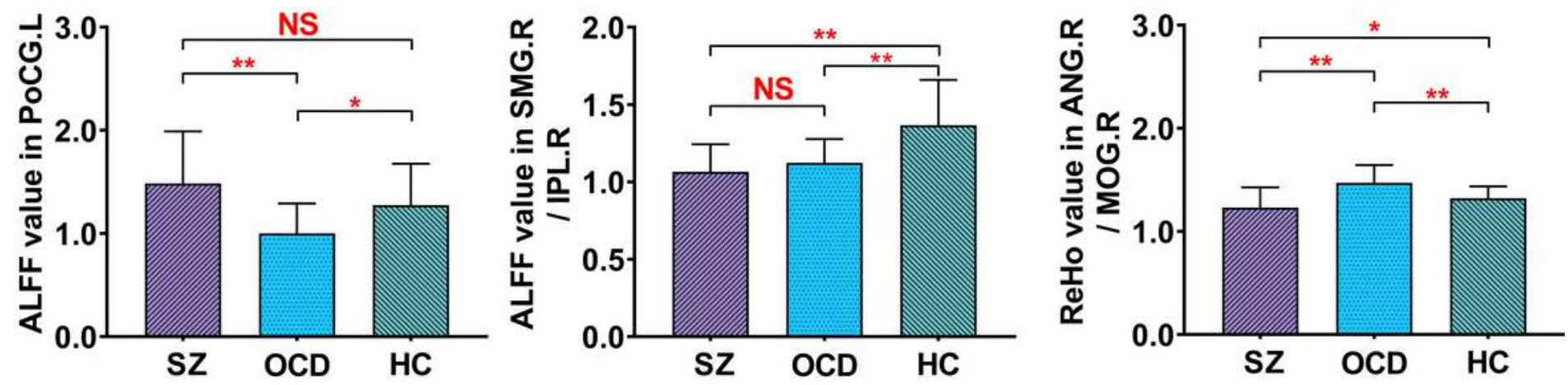

(C)
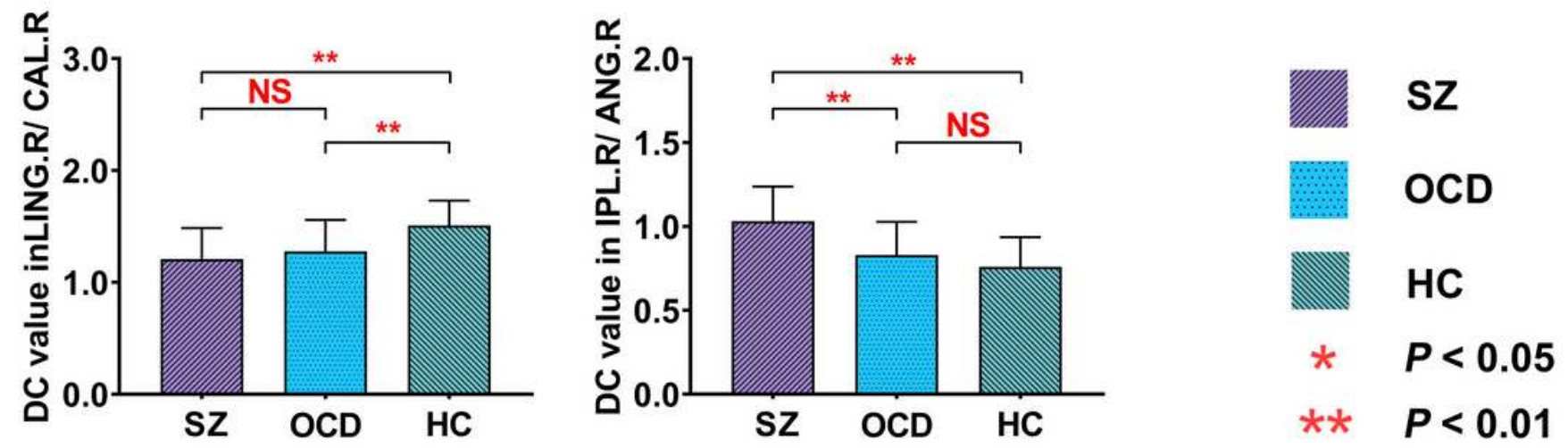

Figure 2

Histogram plots illustrate the mean ALFF/ReHo/DC values of the clusters showing significant 8differences among the healthy controls, patients with schizophrenia and obsessive-compulsive disorder. 
(A). The mean ALFF values in the ANG.R, STG.L/INS.L/ROL.R, MFG.L/PreCG.L, PoCG.L, and SMG.R/IPL.R among the three groups. (B). The mean ReHo values in the ANG.R/MoG.R among the three groups. (C). The mean DC values in the ING.R/CAL.R and IPL.R/ANG.R. Error bars reflect the SD. Abbreviations: HC, healthy controls; OCD, patients with obsessive-compulsive disorder; SZ, patient with schizophrenia; ALFF, amplitude of low-frequency fluctuation; ReHo, regional homogeneity; DC, degree centrality; ANG.R, right angular gyrus; STG.L/INS.L/ROL.R, left superior temporal gyrus/insula/rolandic operculum;

MFG.L/PreCG.L, left middle frontal gyrus/precentral gyrus; PoCG.L, left postcentral gyrus, SMG.R/IPL.R, right supramarginal gyrus/inferior parietal lobule; ANG.R/MoG.R, right angular gyrus/middle occipital gyrus; ING.R/CAL.R, right lingual gyrus/ calcarine fissure and surrounding cortex; IPL.R/ANG.R, right inferior parietal lobule/ angular gyrus.

(A)

(B)
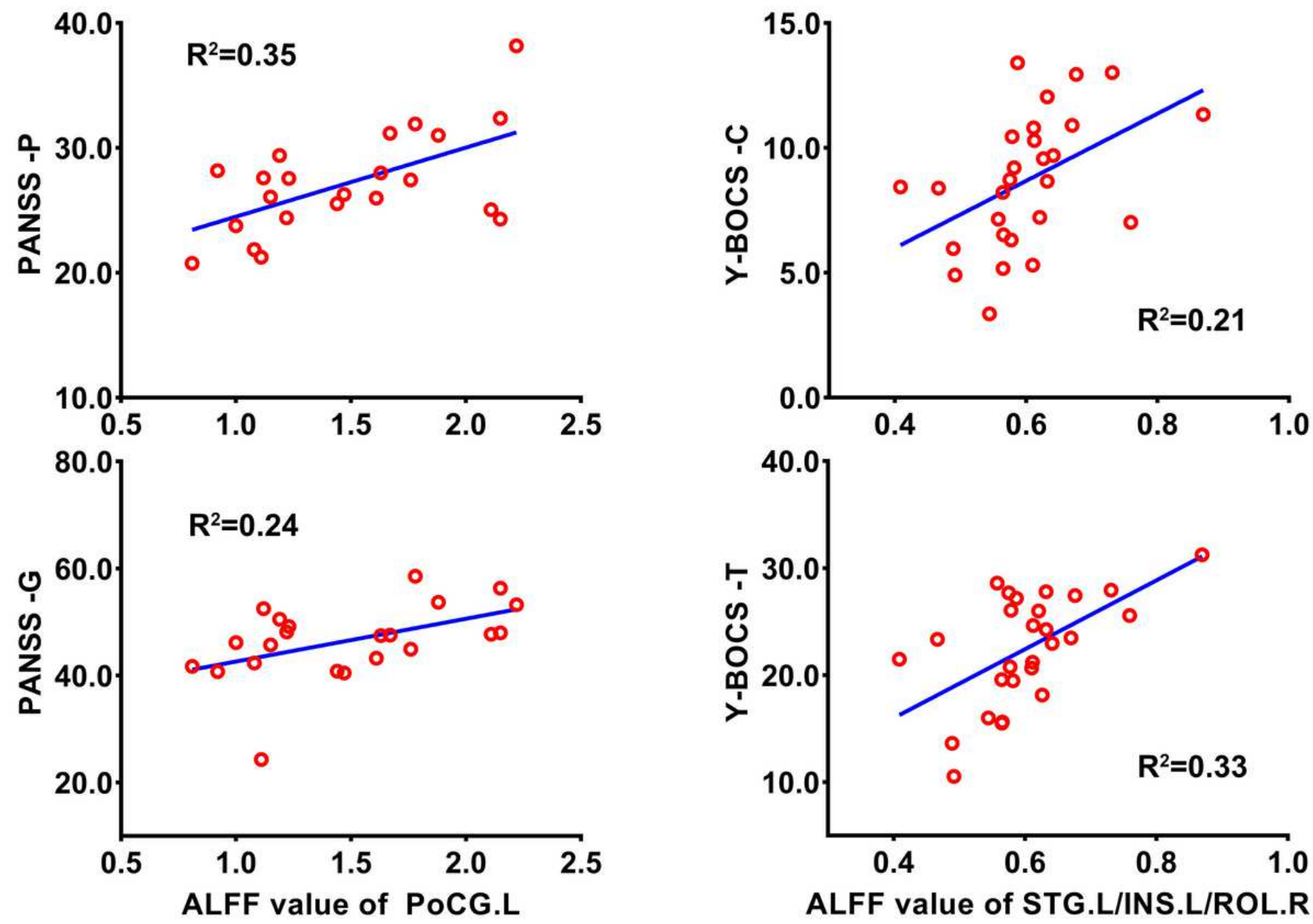

Figure 3

Scatter plots show the relationships between the ALFF values and the clinical symptoms in patients with schizophrenia and obsessive-compulsive disorder. (A). The ALFF values of the left postcentral gyrus of patients with SZ were positively correlated with the scores of PANSS general pathology scale and positive 
subscale; (B). The ALFF values in the left superior temporal gyrus/insula/rolandic operculum brain area of patients with OCD were positively correlated with compulsion subscale scores and the total scores of Y-BOCS. Abbreviations: PANSS, Positive and Negative Syndrome Scale; Y-BOCS, the Yale-Brown Obsessive-Compulsive Scale; ALFF, amplitude of low-frequency fluctuation; PANSS-P, PANSS positive score; PANSS-G, PANSS general psychopathological score; Y-BOCS -C, Y-BOCS compulsive score; YBOCST, Y-BOCS total score; PoCG.L, left postcentral gyrus; STG.L/INS.L/ROL.R, left superior temporal gyrus/insula/rolandic operculum; R2, the coefficient of determination. 